\title{
PROJECTIVE VARIETIES OF MAXIMAL SECTIONAL REGULARITY
}

\author{
MARKUS BRODMANN, WANSEOK LEE, EUISUNG PARK, PETER SCHENZEL
}

\begin{abstract}
We study projective varieties $X \subset \mathbb{P}^{r}$ of dimension $n \geq 2$, of codimension $c \geq 3$ and of degree $d \geq c+3$ that are of maximal sectional regularity, i.e. varieties for which the Castelnuovo-Mumford regularity $\operatorname{reg}(\mathcal{C})$ of a general linear curve section is equal to $d-c+1$, the maximal possible value (see [10]). As one of the main results we classify all varieties of maximal sectional regularity. If $X$ is a variety of maximal sectional regularity, then either (a) it is a divisor on a rational normal $(n+1)$-fold scroll $Y \subset \mathbb{P}^{n+3}$ or else (b) there is an $n$-dimensional linear subspace $\mathbb{F} \subset \mathbb{P}^{r}$ such that $X \cap \mathbb{F} \subset \mathbb{F}$ is a hypersurface of degree $d-c+1$. Moreover, suppose that $n=2$ or the characteristic of the ground field is zero. Then in case (b) we obtain a precise description of $X$ as a birational linear projection of a rational normal $n$-fold scroll.
\end{abstract}

\section{INTRODUCTION}

Let $X \subset \mathbb{P}^{r}$ be a nondegenerate irreducible projective variety of dimension $n$, codimension $c>1$ and degree $d$ over an algebraically closed field $\mathbb{k}$. D. Mumford[15] has defined $X$ to be $m$-regular if its ideal sheaf $\mathcal{I}_{X}$ satisfies the following vanishing condition

$$
H^{i}\left(\mathbb{P}^{r}, \mathcal{I}_{X}(m-i)\right)=0 \text { for all } i \geq 1 .
$$

The $m$-regularity condition implies the $(m+1)$-regularity condition, so that one defines the Castelnuovo-Mumford regularity $\operatorname{reg}(X)$ of $X$ as the least integer $m$ such that $X$ is $m$-regular. It is well known that if $X$ is $m$-regular then its homogeneous ideal is generated by forms of degree $\leq m$. This algebraic implication of $m$-regularity has an elementary geometric consequence that any $(m+1)$-secant line to $X$ should be contained in $X$. We say that a linear space $L \subset \mathbb{P}^{r}$ is $k$-secant to $X$ if

$$
\text { length }(\mathrm{X} \cap \mathrm{L}):=\operatorname{dim}_{\mathbb{k}}\left(\mathcal{O}_{\mathbb{P}^{r}} / \mathcal{I}_{\mathrm{X}}+\mathcal{I}_{\mathrm{L}}\right) \geq \mathrm{k} \text {. }
$$

A well known conjecture due to Eisenbud and Goto (see [6]) says that

$$
\operatorname{reg}(X) \leq d-c+1 .
$$

Obviously this conjecture implies the following conjecture

$$
X \text { has no proper } k \text {-secant line if } k>d-c+1 \text {. }
$$

So far the conjecture (1.1) has been proved only for irreducible but not necessarily smooth curves by Gruson-Lazarsfeld-Peskine[10] and for smooth complex surfaces by $\mathrm{H}$. Pinkham[20] and R. Lazarsfeld[14]. Moreover, in [10] the curves in $\mathbb{P}^{r}$ whose regularity takes the maximally possible value $d-r+2$ are completely classified: they are either

Date: Seoul, 06. January 2015.

2010 Mathematics Subject Classification. Primary: 14H45, 13D02.

Key words and phrases. Castelnuovo-Mumford regularity, variety of maximal sectional regularity, extremal secant locus. 
of degree $\leq r+1$ or else smooth rational curves having a $(d-r+2)$-secant line. The statement (1.2) is known to be true when $X$ is locally Cohen-Macaulay (see Theorem 1 in [17]). But it is still unknown for arbitrary varieties.

The main subject of the present paper is to study the geometry of proper $(d-c+1)$ secant lines to a projective variety. To this aim, we investigate the extremal secant locus $\Sigma(X)$ of $X$, that is, the closure of the set of all proper $(d-c+1)$-secant lines to $X$ in the Grassmannian $\mathbb{G}\left(1, \mathbb{P}^{r}\right)$. Of course, if the extremal secant locus of $X$ is nonempty then its regularity is at least $d-c+1$ and so such a variety will play an important role in the natural problem of classifying all extremal varieties with respect to the above regularity conjecture. For $d \geq c+3$, Gruson-Lazarsfeld-Peskine's result in [10] provides a complete classification of curves having a $(d-c+1)$-secant line. They should be smooth and rational. M. A. Bertin[1] generalizes this result to higher dimensional smooth varieties. She proves the conjecture (1.1) for smooth rational scrolls - which is reproved in [13] and shows that if $X$ is a smooth variety having a $(d-c+1)$-secant line then it should be the linear regular projection of a smooth rational normal scroll. Later, A. Noma[17] obtains a very nice description of those smooth rational scrolls.

In Theorem 3.4 we show that if $c \geq 3$ and $d \geq c+3$, then the dimension of $\Sigma(X)$ is at most $2 n-2$ and the equality is attained if and only if a general linear curve section of $X$ has the maximal Castelnuovo-Mumford regularity $d-c+1$. We will say that $X$ is a variety of maximal sectional regularity if its general linear curve section is of maximal regularity (cf. [4]).

To complete the result starting with Theorem 3.4, it is natural to ask for a classification of all varieties of maximal sectional regularity. This is the contents of Theorem 6.3 and Theorem 7.1. More precisely, for $c \geq 3$ and $d \geq c+3$ we obtain a classification of surfaces of maximal sectional regularity in Theorem 6.3 and a classification of higher dimensional varieties of maximal sectional regularity in Theorem 7.1 . It turns out that $X \subset \mathbb{P}^{r}$ is variety of maximal sectional regularity if and only if it is one of the followings:

(a) $c=3$ and $X$ is a divisor of the $(n+1)$-fold scroll $Y=S(\underbrace{0, \ldots, 0}_{(n-2) \text {-times }}, 1,1,1) \subset \mathbb{P}^{n+3}$ such that $X$ is linearly equivalent to $H+(d-3) F$, where $H$ is the hyperplane divisor of $Y$ and $F \subset Y$ is a linear subspace of dimension $n$;

(b) There exists an $n$-dimensional linear subspace $\mathbb{F} \subset \mathbb{P}^{r}$ such that $X \cap \mathbb{F}$ in $\mathbb{F}$ is a hypersurface of degree $d-c+1$.

In particular, there exist varieties $X \subset \mathbb{P}^{r}$ of maximal sectional regularity of dimension $n$, of codimension $c$ and of degree $d$ for any given $(n, c, d)$ with $n \geq 2, c \geq 3$ and $d \geq c+3$. Furthermore, assume that $\operatorname{char}(\mathbb{k})=0$ or $n=2$. Then in case (b), we obtain a very precise description of $X$ as a birational linear projection of a rational normal $n$-fold scroll. See Theorem 6.3 and Theorem 7.1.

1.1. Remark. Let $X \subset \mathbb{P}^{r}$ be a nondegenerate irreducible projective variety of dimension $n \geq 2$, codimension $c \geq 2$ and degree $d$. Thus $d \geq c+1$.

(1) Our subject of the present paper is quite well understood if $d \leq c+2$. More precisely, varieties of minimal degree (i.e. $d=c+1$ ) are characterized by the 2-regularity (cf. [6]). Of course, these varieties have many proper secant lines. If $d=c+2$, then $\operatorname{reg}(\mathrm{X})=3$ but $X$ may be cut out by quadrics and so it may have no tri-secant lines (cf. [11], [18]). 
(2) One can naturally ask whether $X$ satisfies the regularity bound in (1.1) when it has a nonempty extremal secant locus and hence $\operatorname{reg}(\mathrm{X}) \geq \mathrm{d}-\mathrm{c}+1$. By M. A. Bertin's work in [1], the answer for this question is "YES" when $X$ is smooth. But it is unknown if $X$ is a singular variety. In this direction, the authors in [2] study various cohomological and homological properties of $X$ when it is a surface of maximal sectional regularity. In particular, it is shown that such a surface achieves the regularity bound in (1.1).

\section{Curves of maximal Regularity}

In this section we prove some results on curves of maximal regularity, which will be useful for our later investigations. We first fix a few notations which we shall keep for the rest of this paper.

2.1. Notation and Remarks. Let $\mathcal{C} \subset \mathbb{P}^{r}$ be a nondegenerate projective integral curve of degree $d$.

(A) In their fundamental paper (cf. [10]) Gruson, Lazarsfeld and Peskine have shown that

$$
\operatorname{reg}(\mathcal{C}) \leq d-r+2
$$

and the equality is attained if and only if either $d \leq r+1$ or else $d \geq r+2$ and $\mathcal{C}$ is a smooth rational curve having a $(d-r+2)$-secant line. We say that $\mathcal{C}$ is of maximal regularity if $\operatorname{reg}(\mathcal{C})=d-r+2$.

(B) If $d \geq r+2$ and $\mathcal{C}$ is a curve of maximal regularity, then $\mathcal{C}$ is the regular projection of a rational normal curve $\widetilde{\mathcal{C}} \subset \mathbb{P}^{d}$ of degree $d$ and hence $\mathcal{C}$ is not linearly normal.

(C) Let $r \geq 4$ and $d \geq r+2$. According to [3, Remark 3.1(C)], if $\mathcal{C}$ is of maximal regularity then the $(d-r+2)$-secant line of statement $(\mathrm{A})$ is uniquely determined. We will denote this line by $\mathbb{L}_{\mathcal{C}}$. Throughout this section we will see that this line induces additional geometric properties of $\mathcal{C}$.

2.2. Notation and Remarks. We recall a standard description of rational normal scrolls (cf. [21]). For the vector bundle

$$
\mathcal{E}=\mathcal{O}_{\mathbb{P}^{1}}\left(a_{1}\right) \oplus \cdots \oplus \mathcal{O}_{\mathbb{P}^{1}}\left(a_{n}\right)
$$

on $\mathbb{P}^{1}$ where $0 \leq a_{1} \leq \cdots \leq a_{n}$ and $a_{n}>0$, the tautological line bundle $\mathcal{O}_{\mathbb{P}(\mathcal{E})}(1)$ of $\mathbb{P}(\mathcal{E})$ is globally generated and we write $S\left(a_{1}, \cdots, a_{n}\right)$ for the image of the map defined by $\mathcal{O}_{\mathbb{P}(\mathcal{E})}(1)$.

(A) It is well-known that $S\left(a_{1}, \cdots, a_{n}\right)$ is a normal variety and has only rational singularities. Also the homogeneous ideal of $S\left(a_{1}, \cdots, a_{n}\right)$ is generated by quadrics. In particular, any tri-secant line to $S\left(a_{1}, \cdots, a_{n}\right)$ is contained in $S\left(a_{1}, \cdots, a_{n}\right)$.

(B) Suppose that $n \geq 2$. Then the divisor class group of $\mathbb{P}(\mathcal{E})$ is freely generated by $\widetilde{H} \in\left|\mathcal{O}_{\mathbb{P}(\mathcal{E})}(1)\right|$ and a linear subspace $\widetilde{F}$ of dimension $n-1$. Moreover, if $a_{n-1}>0$ then the morphism $\varphi: \mathbb{P}(\mathcal{E}) \rightarrow S\left(a_{1}, \cdots, a_{n}\right)$ induces an isomorphism between the divisor class groups. Thus the divisor class group of $S\left(a_{1}, \cdots, a_{n}\right)$ is freely generated by the hyperplane divisor $H$ and a linear subspace $F$ of dimension $n-1$. We refer the reader to [7].

(C) One can compute explicitly the dimension of $H^{i}\left(\mathbb{P}(\mathcal{E}), \mathcal{O}_{\mathbb{P}(\mathcal{E})}(a \widetilde{H}+b \widetilde{F})\right)$ by using the projective bundle map $j: \mathbb{P}(\mathcal{E}) \rightarrow \mathbb{P}^{1}$. For example, see [16, Section 2]. 
2.3. Proposition. Let $r \geq 4$ and $d \geq r+2$. Let $\mathcal{C} \subset \mathbb{P}^{r}$ be a curve of degree $d$ which is of maximal regularity and let $\mathbb{L}_{\mathcal{C}}$ be as in Notation and Remarks 2.1(B). Then

(a) $\operatorname{Join}\left(\mathbb{L}_{\mathcal{C}}, \mathcal{C}\right)$ is projectively equivalent to the threefold scroll $S(0,0, r-2)$.

(b) Suppose that $\mathcal{C}$ is contained in a rational normal threefold scroll $Y:=S(0,0, r-2)$. Then $Y=\operatorname{Join}\left(\mathbb{L}_{\mathcal{C}}, \mathcal{C}\right)$ and the vertex $\mathbb{L}=S(0,0) \subset S(0,0, r-2)$ of $Y$ is equal to $\mathbb{L}_{\mathcal{C}}$. In particular, Join $\left(\mathbb{L}_{\mathcal{C}}, \mathcal{C}\right)$ is the only rational normal threefold scroll which is projectively equivalent to $S(0,0, r-2)$ and which contains $\mathcal{C}$.

Proof. (a): Choose a subspace $\mathbb{P}^{r-2} \subset \mathbb{P}^{r}$ which does not meet $\mathbb{L}_{\mathcal{C}}$ and consider the linear projection map

$$
\pi_{\mathbb{L}_{\mathcal{C}}}: \mathbb{P}^{r} \backslash \mathbb{L}_{\mathcal{C}} \rightarrow \mathbb{P}^{r-2}
$$

of $\mathcal{C}$ from $\mathbb{L}_{\mathcal{C}}$. Then $\mathcal{C}^{\prime}:=\overline{\pi_{\mathbb{L}_{\mathcal{C}}}\left(\mathcal{C} \backslash \mathbb{L}_{\mathcal{C}}\right)}$ is a nondegenerate rational normal curve in $\mathbb{P}^{r-2}$ and $\operatorname{Join}\left(\mathbb{L}_{\mathcal{C}}, \mathcal{C}\right)$ is the cone over $\mathcal{C}^{\prime}$ with vertex $\mathbb{L}_{\mathcal{C}}$.

(b): We assume that $\mathbb{L} \neq \mathbb{L}_{\mathcal{C}}$ and aim for a contradiction. As length $\left(\mathcal{C} \cap \mathbb{L}_{\mathcal{C}}\right)>2$ we have $\mathbb{L}_{\mathcal{C}} \subset Y$ and hence $\left\langle\mathbb{L}, \mathbb{L}_{\mathcal{C}}\right\rangle \subset Y$, so that $\mathbb{L}$ and $\mathbb{L}_{\mathcal{C}}$ are coplanar. Now, consider the linear projection map $\pi_{\mathbb{L}}: \mathbb{P}^{r} \backslash \mathbb{L} \rightarrow \mathbb{P}^{r-2}$. The restriction map $\pi_{\mathbb{L}} \uparrow: Y \backslash \mathbb{L} \rightarrow S(r-2)$ induces a dominant morphism $\mathcal{C} \backslash(\mathcal{C} \cap \mathbb{L}) \rightarrow S(r-2)$. As $\mathcal{C}$ is smooth, this morphism may be extended to a surjective morphism $\phi: \mathcal{C} \rightarrow S(r-2)$. This implies that

$$
\operatorname{deg}(\phi)=\frac{d-\operatorname{length}(\mathcal{C} \cap \mathbb{L})}{\operatorname{deg}_{\mathbb{P}^{r-2}}(S(r-2))} \leq \frac{d}{r-2} .
$$

As $\mathbb{L}$ and $\mathbb{L}_{\mathcal{C}}$ are coplanar, $\phi\left(\mathcal{C} \cap \mathbb{L}_{\mathcal{C}}\right)=\pi_{\mathbb{L}}\left(\mathbb{L}_{\mathcal{C}} \backslash \mathbb{L}\right)$ is a point, say $z \in S(r-2)$. As $S(r-2)$ is smooth, this implies that

$$
\operatorname{deg}(\phi)=\operatorname{length}\left(\phi^{-1}(\mathrm{z})\right) \geq \operatorname{length}\left(\mathcal{C} \cap \mathbb{L}_{\mathcal{C}}\right)=\mathrm{d}-\mathrm{r}+2 .
$$

The two previous inequalities imply that $\frac{d}{r-2} \geq d-r+2$, which is impossible since $d \geq r+2$. This contradiction shows that $\mathbb{L}=\mathbb{L}_{\mathcal{C}}$ and hence proves our claim.

Let $\mathcal{C} \subset \mathbb{P}^{r}$ be as in the above Proposition 2.3. In the next Proposition 2.4, we study the case where our curve $\mathcal{C}$ lies on a smooth rational normal surface. Note that the threefold scroll Join $\left(\mathbb{L}_{\mathcal{C}}, \mathcal{C}\right)=S(0,0, r-2)$ contains many smooth rational normal surface scrolls projectively equivalent to $S(1, r-2)$. For example, any isomorphism from $\mathbb{L}_{\mathcal{C}}=S(0,0)$ to $\mathcal{C}^{\prime}$ in the above proof defines a rational normal surface scroll of type $S(1, r-2)$ which is contained in $\operatorname{Join}\left(\mathbb{L}_{\mathcal{C}}, \mathcal{C}\right)$. Also it may happen that $\mathcal{C}$ is contained in such a surface scroll.

2.4. Proposition. Let $\mathcal{C} \subset \mathbb{P}^{r}$ be as in Proposition 2.3. If $\mathcal{C}$ is contained in a smooth rational normal surface scroll $S=S(\alpha, r-\alpha-1)$ for some $1 \leq \alpha \leq \frac{r-1}{2}$, then

(a) $\alpha=1$,

(b) $\mathcal{C}$ is linearly equivalent to the divisor $H+(d-r+1) F$ where $H$ and $F$ respectively are a general hyperplane section and a ruling line of $S$,

(c) $\mathbb{L}_{\mathcal{C}}$ is equal to the unique line section $S(1) \subset S(1, r-2)$ of $S$, and

(d) $S$ is contained in $\operatorname{Join}\left(\mathbb{L}_{\mathcal{C}}, \mathcal{C}\right)$.

Proof. Let $\mathcal{C}$ be linearly equivalent to $a H+b F$. Then $a \geq 1$ since $\mathcal{C}$ is irreducible and not a line. As the surface $S$ is arithmetically Cohen-Macaulay, we have $H^{i}\left(\mathbb{P}^{r}, \mathcal{I}_{S}(1)\right)=0$ for 
$i=1,2$ and so, the short exact sequence

$$
0 \longrightarrow \mathcal{I}_{S} \longrightarrow \mathcal{I}_{\mathcal{C}} \longrightarrow \mathcal{O}_{S}(-\mathcal{C}) \longrightarrow 0
$$

implies an isomorphism $H^{1}\left(\mathbb{P}^{r}, \mathcal{I}_{\mathcal{C}}(1)\right) \cong H^{1}\left(S, \mathcal{O}_{S}((1-a) H-b F)\right)$. If $a>1$, we have $H^{1}\left(\mathbb{P}^{r}, \mathcal{O}_{S}((1-a) H-b F)\right)=0$, and we get the contradiction that $\mathcal{C} \subset \mathbb{P}^{r}$ is linearly normal (cf. Notation and Remarks 2.1(C)). Therefore $a=1$. As $d=\operatorname{deg}(\mathcal{C})=\operatorname{deg}(S)+b=$ $r-1+b$, we obtain $b=d-r+1$ and $\mathcal{C}$ is linearly equivalent to $H+(d-r+1) F$.

Now, we will see that $\alpha=1$. Note that $S$ is cut out by quadrics and so $\mathbb{L}_{\mathcal{C}}$ should be contained in $S$ (cf. Notation and Remarks 2.2(A)). If $\alpha \geq 2$ then the only lines contained in $S$ are the ruling lines. Obviously, no ruling line of $S$ can be a multi-secant line to $\mathcal{C}$. Therefore $\alpha=1$.

The unique line section $\mathbb{L}=S(1)$ of $S=S(1, r-2)$ satisfies the condition

$$
\text { length }(\mathcal{C} \cap \mathbb{L})=\mathcal{C} \cdot \mathbb{L}=(\mathrm{H}+(\mathrm{d}-\mathrm{r}+1) \mathrm{F}) \cdot(\mathrm{H}-(\mathrm{r}-2) \mathrm{F})=\mathrm{d}-\mathrm{r}+2,
$$

and hence $\mathbb{L}$ is indeed the unique $(d-r+2)$-secant line to $\mathcal{C}$. Now, it is clear that $S$ is contained in the threefold scroll $\operatorname{Join}(S(1), \mathcal{C})=\operatorname{Join}\left(\mathbb{L}_{\mathcal{C}}, \mathcal{C}\right)$.

\section{The EXTREmal SECANT LOCUs of A PROJECTIVE VARIETY}

In this section, we study the geometry of proper $(d-c+1)$-secant lines to a nondegenerate irreducible projective variety $X \subset \mathbb{P}^{r}$ of codimension $c$ and degree $d$. To this aim, we will investigate the extremal secant locus $\Sigma(X)$ of $X$, that is, the closure of the set of all proper $(d-c+1)$-secant lines of $X$ in the Grassmannian $\mathbb{G}\left(1, \mathbb{P}^{r}\right)$.

To give precise statements, we require some notation and definitions. We first fix a few notations, which we shall keep for the rest of our paper.

3.1. Notation and Reminder. Let $X \subset \mathbb{P}^{r}$ be as above.

(A) Let $\Sigma_{m}(X)$ be the locus of all $m$-secant lines of $X$ in $\mathbb{G}\left(1, \mathbb{P}^{r}\right)$. That is,

$$
\Sigma_{m}(X):=\left\{\mathbb{L} \in \mathbb{G}\left(1, \mathbb{P}^{r}\right) \mid \operatorname{length}(\mathrm{X} \cap \mathbb{L}) \geq \mathrm{m}\right\} .
$$

This set is closed in $\mathbb{G}\left(1, \mathbb{P}^{r}\right)$. We also shall use the notation

$$
\Sigma_{\infty}(X):=\left\{\mathbb{L} \in \mathbb{G}\left(1, \mathbb{P}^{r}\right) \mid \mathbb{L} \subseteq X\right\} .
$$

Thus we have the inclusion $\Sigma_{\infty}(X) \subseteq \Sigma_{m}(X)$ and the equality holds if $X$ is cut out by forms of degree $<m$. In particular, $\Sigma_{\infty}(X)$ is a closed subset, too.

(B) The set $\Sigma_{m}^{\circ}(X):=\Sigma_{m}(X) \backslash \Sigma_{\infty}(X)$ of all proper $m$-secant lines to $X$ is locally closed in $\mathbb{G}\left(1, \mathbb{P}^{r}\right)$. We define $\mathfrak{d}_{m}(X)$ and $\overline{\mathfrak{d}}_{m}(X)$ respectively as

$$
\mathfrak{d}_{m}(X):=\operatorname{dim} \Sigma_{m}(X) \text { and } \overline{\mathfrak{d}}_{m}(X):=\operatorname{dim} \overline{\Sigma_{m}^{\circ}(X)}\left(=\operatorname{dim} \Sigma_{m}^{\circ}(X)\right) .
$$

(C) By definition, the extremal secant locus $\Sigma(X)$ of $X$ is equal to $\overline{\Sigma_{d-c+1}^{\circ}(X)}$.

(D) Recall that in the introduction we define $X$ to be a variety of maximal sectional regularity if the general linear curve section of $X$ is of maximal regularity. To be precise, $X$ is of maximal sectional regularity if there exists a nonempty open subset $\mathcal{U} \subset \mathbb{G}\left(c+1, \mathbb{P}^{r}\right)$ such that 
(*) For any $\Lambda \in \mathcal{U}$, the intersection

$$
\mathcal{C}_{\Lambda}:=X \cap \Lambda \quad \subset \quad \Lambda=\mathbb{P}^{c+1}
$$

is an integral curve of maximal regularity.

In this case, we will denote by $\mathcal{U}(X)$ the largest open subset of $\mathbb{G}\left(c+1, \mathbb{P}^{r}\right)$ satisfying the property $(*)$.

Now, we are heading for the main result of this section. We begin with the following auxiliary result.

3.2. Lemma. Let $T$ be an integral closed subset of $\mathbb{G}\left(1, \mathbb{P}^{r}\right)$ and let $\mathbb{H}=\mathbb{P}^{r-1} \subset \mathbb{P}^{r}$ be a general hyperplane. Then, the following statements hold.

(a) If $\operatorname{dim} T \leq 1$, then $T \cap \mathbb{G}(1, \mathbb{H})=\emptyset$.

(b) If $\operatorname{dim} T \geq 2$, then each irreducible component $W$ of $T \cap \mathbb{G}(1, \mathbb{H})$ satisfies

$$
\operatorname{dim} W=\operatorname{dim} T-2 .
$$

Proof. Fix a hyperplane $\mathbb{H}_{0} \subset \mathbb{P}^{r}$. The canonical action of the integral algebraic group scheme $G=$ : $\operatorname{Aut}\left(\mathbb{P}^{r}\right)$ on the integral scheme $X=\mathbb{G}\left(1, \mathbb{P}^{r}\right)$ is transitive. Thus a result of Kleiman (see [12, Corollary 4]) says that all irreducible components of $g\left(\mathbb{G}\left(1, \mathbb{H}_{0}\right)\right) \cap T$ have dimension

$$
\operatorname{dim} \mathbb{G}\left(1, \mathbb{H}_{0}\right)+\operatorname{dim} T-\operatorname{dim} \mathbb{G}\left(1, \mathbb{P}^{r}\right)=\operatorname{dim} T-2
$$

for general $g \in G$.

3.3. Proposition. Let $X \subset \mathbb{P}^{r}$ be a nondegenerate irreducible projective variety of dimension $n$, codimension $c \geq 3$ and degree $d \geq c+3$. Then

(a) $\mathfrak{d}_{\infty}(X) \leq 2 n-3$.

(b) $\mathfrak{d}_{d-c+1}(X) \leq 2 n-2$ and the equality is attained if and only if $X$ is a variety of maximal sectional regularity.

Proof. For any hyperplane $\mathbb{H}$ of $\mathbb{P}^{r}$ and any $m \in \mathbb{N} \cup\{\infty\}$, it holds that

$$
\Sigma_{m}(X \cap \mathbb{H})=\Sigma_{m}(X) \cap \mathbb{G}(1, \mathbb{H}) .
$$

Now, let $\mathbb{H}_{1}, \ldots, \mathbb{H}_{n-1}$ be general hyperplanes and let $\mathcal{C}:=X \cap \mathbb{H}_{1} \cap \cdots \cap \mathbb{H}_{n-1}$. Then by combining Lemma 3.2 and (3.1), one can see that

(i) $\Sigma_{m}(\mathcal{C})$ is empty if and only if $\mathfrak{d}_{m} \leq 2 n-3$ and

(ii) $\mathfrak{d}_{m}(\mathcal{C}) \geq 0$ if and only if $\mathfrak{d}_{m}(X) \geq 2 n-2$. In this case, it holds that

$$
\mathfrak{d}_{m}(\mathcal{C})=\mathfrak{d}_{m}(X)-(2 n-2) .
$$

(a): Obviously, $\Sigma_{\infty}(\mathcal{C})$ is empty. Thus we have $\mathfrak{d}_{\infty}(X) \leq 2 n-3$ by (i).

(b): We know that $\mathcal{C}$ has at most one $(d-c+1)$-secant line and so $\mathfrak{d}_{d-c+1}(\mathcal{C}) \leq 0$ (cf. Notation and Remarks 2.1.(B)). It follows by (i) and (ii) that $\mathfrak{d}_{d-c+1}(X) \leq 2 n-2$. Moreover, $\mathfrak{d}_{d-c+1}(X)=2 n-2$ if and only if $\mathfrak{d}_{d-c+1}(\mathcal{C})=0$ and hence $\mathcal{C}$ is a curve of maximal regularity.

3.4. Theorem. Let $X \subset \mathbb{P}^{r}$ be as in Proposition [3.3. Then $\overline{\mathfrak{d}}_{d-c+1}(X) \leq 2 n-2$ and equality is attained if and only if $X$ is a variety of maximal sectional regularity. 
Proof. Since it holds always that $\mathfrak{d}_{d-c+1}(X) \geq \overline{\mathfrak{d}}_{d-c+1}(X)$, we get the desired inequality $\overline{\mathfrak{d}}_{d-c+1}(X) \leq 2 n-2$ from Proposition [3.3(b). Also since $\mathfrak{d}_{\infty}(X) \leq 2 n-3$ by Proposition 3.3. (a), it holds that $\mathfrak{d}_{d-c+1}(X)=2 n-2$ if and only if $\overline{\mathfrak{d}}_{d-c+1}(X)=2 n-2$.

\section{Sectionally Rational Varieties}

Let $X \subset \mathbb{P}^{r}$ be a nondegenerate irreducible projective variety. We will say that $X$ is a sectionally rational variety (resp. sectionally smooth rational variety) if its general linear curve section is rational (resp. smooth rational). We are interested in this kind of varieties since any variety of maximal sectional regularity is sectionally smooth rational (cf. Notation and Remarks 2.1(A)). The aim of this section is to show - under some mild conditions - that a sectionally rational variety is always obtained as a birational linear projection of a variety of minimal degree.

4.1. Theorem. Let $X \subset \mathbb{P}^{r}$ be a nondegenerate irreducible projective variety of dimension $n$ and degree $d$. Assume that either

(1) $\operatorname{char}(\mathbb{k})=0$ and $X$ is a sectionally rational variety, or else

(2) $X$ is a sectionally smooth rational surface.

Then $X$ is a projection of a variety of minimal degree. More precisely, $X=\pi_{\Lambda}(\tilde{X})$ where

(a) $\widetilde{X} \subset \mathbb{P}^{d+n-1}$ is an $n$-dimensional variety of minimal degree,

(b) $\Lambda=\mathbb{P}^{d+n-r-2} \subset \mathbb{P}^{d+n-1}$ is a subspace such that $\tilde{X} \cap \Lambda=\emptyset$,

(c) $\pi_{\Lambda}: \mathbb{P}^{d+n-1} \backslash \Lambda \rightarrow \mathbb{P}^{r}$ is the linear projection map from $\Lambda$ and

(d) $\pi_{\Lambda} \uparrow: \widetilde{X} \rightarrow X$ is the normalization of $X$.

Furthermore, $X$ is a sectionally smooth rational variety if and only if the singular locus

$$
\operatorname{Sing}\left(\pi_{\Lambda}\right):=\left\{\mathrm{x} \in \mathrm{X} \mid \operatorname{length}\left(\pi_{\Lambda}^{-1}(\mathrm{x})\right) \geq 2\right\}
$$

of $\pi_{\Lambda}: \widetilde{X} \rightarrow X$ has dimension at most $n-2$.

Proof. Let $\nu: Y \rightarrow X$ be the normalization of $X$, so that $Y$ is an $n$-dimensional normal projective variety and $\nu$ is a finite surjective birational morphism. Also the line bundle $\mathcal{L}:=\nu^{*} \mathcal{O}_{X}(1)$ on $Y$ is ample and base point free. Let $\mathbb{H}_{1}, \ldots, \mathbb{H}_{n-1}$ be general hyperplanes and consider the $\ell$-dimensional irreducible varieties $X_{\ell}:=X \cap \mathbb{H}_{1} \cap \cdots \cap \mathbb{H}_{n-\ell}$ and their preimages $Y_{\ell}:=\nu^{-1}\left(X_{\ell}\right) \quad(\ell=1, \ldots n)$. As the hyperplanes $\mathbb{H}_{j}$ are general, $X_{\ell}$ is not contained in the singular locus of $\nu$ and so $Y_{\ell}$ are irreducible and the induced finite morphisms

$$
\nu_{\ell}:=\nu \uparrow: Y_{\ell} \rightarrow X_{\ell}, \quad \ell=1, \ldots, n
$$

are birational. Note that $X_{1}$ is a rational curve since $X$ is sectionally rational.

Assume first that $\operatorname{char}(\mathbb{k})=0$. As $Y_{\ell} \subset Y$ is cut out by the $n-\ell$ general divisors $\nu^{*}\left(X \cap \mathbb{H}_{1}\right), \ldots, \nu^{*}\left(X \cap \mathbb{H}_{n-\ell}\right) \in|\mathcal{L}|$, it is normal by the Bertini Theorem [8, Corollary 3.4.2]. So, the sequence

$$
Y_{1} \subset Y_{2} \subset \ldots \subset Y_{t}=Y
$$

forms a ladder with normal rungs of the polarized variety $(Y, \mathcal{L})$ in the sense of T. Fujita[9]. As $\nu_{1}: Y_{1} \rightarrow X_{1}$ is birational, it follows that $Y_{1} \cong \mathbb{P}^{1}$ and hence the sectional genus $g(Y, \mathcal{L})$ of the polarized variety $(Y, \mathcal{L})$ is equal to zero. 
Assume now, that $X$ is a sectionally smooth rational surface. Thus the curve $X_{1} \subset X$ is smooth rational and $\nu_{1}: Y_{1} \rightarrow X_{1}$ is an isomorphism. Hence, again the polarized surface $(Y, \mathcal{L})$ has a ladder $Y_{1} \subset Y_{2}=Y$ with normal rungs and its sectional genus $g(Y, \mathcal{L})$ is zero.

Thus, in both cases the sectional genera satisfy $g(Y, \mathcal{L})=0$. Therefore, by [9, Proposition 3.4], the $\Delta$-genus $\Delta(Y, \mathcal{L})$ of the polarized variety $(Y, \mathcal{L})$ is equal to zero. According to T. Fujita's Classification Theorem [9, Theorem 5.15], it now follows that $\mathcal{L}$ is a very ample line bundle which embeds $Y$ to the $(d+n-1)$-dimensional projective space as a variety of minimal degree. Let

$$
\widetilde{X} \subset \mathbb{P}^{d+n-1}
$$

be the image of the linearly normal embedding $\varphi_{|\mathcal{L}|}: Y \rightarrow \mathbb{P}^{d+n-1}$. It is clear that the normalization map $\nu: Y \rightarrow X$ consists of the embedding $\varphi_{|\mathcal{L}|}$ of $Y$ followed by a linear projection $\pi_{\Lambda}: \mathbb{P}^{d+n-1} \backslash \Lambda \rightarrow \mathbb{P}^{r}$ from a linear space $\Lambda=\mathbb{P}^{d+n-r-2}$. In particular, the map $\pi_{\Lambda}: \widetilde{X} \rightarrow X$ is the normalization of $X$.

Finally, consider the short exact sequence $0 \rightarrow \mathcal{O}_{X} \rightarrow\left(\pi_{\Lambda}\right)_{*} \mathcal{O}_{\tilde{X}} \rightarrow \mathcal{F} \rightarrow 0$ on $X$ where $\mathcal{F}$ is the quotient sheaf $\left(\pi_{\Lambda}\right)_{*} \mathcal{O}_{\tilde{X}} / \mathcal{O}_{X}$. Note that $\operatorname{Sing}\left(\pi_{\Lambda}\right)=\operatorname{Supp}(\mathcal{F})$. In particular, the dimension of $\operatorname{Sing}\left(\pi_{\Lambda}\right)$ is equal to the degree of the Euler-Poincaré characteristic $\chi(\mathcal{F}(t))$. Let us write $\chi\left(\mathcal{O}_{X}(t)\right)$ and $\chi\left(\mathcal{O}_{\widetilde{X}}(t)\right)$ respectively as

$$
\chi\left(\mathcal{O}_{X}(t)\right)=\sum_{j=0}^{n} \chi_{j}\left(\mathcal{O}_{X}(1)\right)\left(\begin{array}{c}
t+j-1 \\
j
\end{array}\right) \quad \text { and } \quad \chi\left(\mathcal{O}_{\tilde{X}}(t)\right)=\sum_{j=0}^{n} \chi_{j}\left(\mathcal{O}_{\tilde{X}}(1)\right)\left(\begin{array}{c}
t+j-1 \\
j
\end{array}\right) .
$$

Here, it holds that $\chi_{n}\left(\mathcal{O}_{X}(1)\right)=\chi_{n}\left(\mathcal{O}_{\widetilde{X}}(1)\right)=d$ since $\pi_{\Lambda}: \widetilde{X} \rightarrow X$ is a finite birational morphism. Also $\chi_{n-1}\left(\mathcal{O}_{\widetilde{X}}(1)\right)=1$ since the general linear curve section of $\widetilde{X}$ is a smooth rational curve. Now, let $m$ be the degree of the polynomial $\chi(\mathcal{F}(t))$. Then, from the relation $\chi(\mathcal{F}(t))=\chi\left(\mathcal{O}_{\tilde{X}}(t)\right)-\chi\left(\mathcal{O}_{X}(t)\right)$ among the Euler-Poincaré characteristics, we can see that $m \leq n-2$ if and only if $\chi_{n-1}\left(\mathcal{O}_{X}(1)\right)=1$ and hence the general linear curve section of $X$ is a curve of arithmetic genus 0 , or equivalently, a smooth rational curve.

It occurs to me, that Corollary 4.2 could be extended as follows:

Assume that $X \subset \mathbb{P}^{r}$ is as in Theorem 4.1 and that $\mathrm{X}$ has only finitely many non-normal points (which is always the case if condition (2) of Theorem 4.1 holds). Then

$$
\chi\left(\mathcal{O}_{X}(t)\right)=d\left(\begin{array}{c}
t+n-1 \\
n
\end{array}\right)+\left(\begin{array}{c}
t+n-1 \\
n-1
\end{array}\right)-\delta(X)
$$

where

$$
\delta(X)=\operatorname{length}\left(\left(\pi_{\Lambda}\right)_{*} \mathcal{O}_{\widetilde{X}} / \mathcal{O}_{X}\right) .
$$

4.2. Corollary. Let $X \subset \mathbb{P}^{r}$ be a sectionally smooth rational surface of degree $d$. Then

$$
\chi\left(\mathcal{O}_{X}(t)\right)=d\left(\begin{array}{c}
t+1 \\
2
\end{array}\right)+t+1-\delta(X)
$$

for a non-negative integer $\delta(X)$. Furthermore, $X$ is smooth if and only if $\delta(X)=0$. 
Proof. From Theorem 4.1 and its proof, we have $\chi\left(\mathcal{O}_{X}(t)\right)=\chi\left(\mathcal{O}_{\widetilde{X}}(t)\right)-\chi(\mathcal{F}(t))$. Also $\operatorname{Supp}(\mathcal{F})$ is at most a finite set and hence $\chi(\mathcal{F}(t))$ is a non-negative integer, say $\delta(X)$. Since

$$
\chi\left(\mathcal{O}_{\widetilde{X}}(t)\right)=d\left(\begin{array}{c}
t+1 \\
2
\end{array}\right)+t+1
$$

we get the desired formula of $\chi\left(\mathcal{O}_{X}(t)\right)$. Moreover, $\delta(X)=0$ if and only if $\pi_{\Lambda}: \widetilde{X} \rightarrow \mathbb{P}^{r}$ is an isomorphic projection of $\widetilde{X}$. Since $\widetilde{X}$ is either smooth or else a cone, we can rephrase this fact as $\delta(X)=0$ if and only if $X$ is smooth.

4.3. Corollary. Let $X \subset \mathbb{P}^{r}$ be a nondegenerate irreducible projective variety of dimension $n$ and degree $d$ which is of maximal sectional regularity. If $\operatorname{char}(\mathbb{k})=0$ or if $n=2$, then $X$ is a projection of a rational normal scroll. More precisely, $X=\pi_{\Lambda}(\tilde{X})$ where

(a) $\widetilde{X} \subset \mathbb{P}^{d+n-1}$ is a rational normal $n$-fold scroll,

(b) $\Lambda=\mathbb{P}^{d+n-r-2} \subset \mathbb{P}^{d+n-1}$ is a subspace with $\widetilde{X} \cap \Lambda=\emptyset$,

(c) $\pi_{\Lambda}: \mathbb{P}^{d+n-1} \backslash \Lambda \rightarrow \mathbb{P}^{r}$ is the linear projection map from $\Lambda$ and

(d) $\pi_{\Lambda} \uparrow: \widetilde{X} \rightarrow X$ is the normalization of $X$.

Furthermore, if $X$ is not a cone then $\tilde{X}$ is a smooth rational normal scroll.

Proof. Since $X$ is sectionally smooth rational (cf. Notation and Remarks 2.1(A)), it holds by Theorem 4.1 that $X$ is a projection of a variety of minimal degree. In our case, $d$ is at least 5 and hence the projecting variety $\widetilde{X}$ should be an $n$-fold rational normal scroll.

If $X$ is not a cone, then $\widetilde{X}$ should be not a cone and hence smooth by the well-known classification result of varieties of minimal degree.

\section{THE EXTREMAL VARIETY OF A VARIETY OF MAXIMAL SECTIONAL REGULARITY}

Let $X \subset \mathbb{P}^{r}$ be a nondegenerate irreducible projective variety of dimension $n$, codimension $c \geq 3$ and degree $d \geq c+3$. Assume that $X$ is of maximal sectional regularity and let $\mathcal{U}(X) \subset \mathbb{G}\left(c+1, \mathbb{P}^{r}\right)$ be as in Notation and Reminder 3.1(D). Then for any $\Lambda \in \mathcal{U}(X)$, the intersection

$$
\mathcal{C}_{\Lambda}:=X \cap \Lambda \subset \mathbb{P}^{c+1}
$$

is an integral curve of maximal regularity. In particular, it admits a unique $(d-c+1)$ secant line, say $\mathbb{L}_{\Lambda}$ (cf. Notation and Remarks 2.1(B)). Along this line, we consider the extremal variety $\mathbb{F}(X)$ of $X$ which is defined as

$$
\mathbb{F}(X)=\overline{\bigcup_{\Lambda \in \mathcal{U}(X)} \mathbb{L}_{\Lambda}} \subset \mathbb{P}^{r} .
$$

Through the next two sections it will turn out that either $\mathbb{F}(X)$ is an $n$-dimensional linear space or else $c=3$ and $\mathbb{F}(X)$ is the $(n+1)$-fold rational normal scroll

$$
S(\underbrace{0, \ldots, 0}_{(n-2) \text {-times }}, 1,1,1) \subset \mathbb{P}^{n+3} .
$$

This structure of the extremal variety will play a crucial role in the classification of varieties of maximal sectional regularity. 
Along this line, this section is devoted to prove a criterion on the linearity of $\mathbb{F}(X)$ and to classify varieties of maximal sectional regularity whose extremal variety is a linear space.

5.1. Lemma. Let $X \subset \mathbb{P}^{r}$ be a nondegenerate irreducible projective variety of dimension $n$, codimension $c \geq 3$ and degree $d \geq c+3$. Suppose that there exists an $n$-dimensional linear subspace $\mathbb{F} \subset \mathbb{P}^{r}$ such that $\operatorname{dim}(X \cap \mathbb{F})=n-1$.

(a) If $\operatorname{deg}_{\mathbb{F}}(X \cap \mathbb{F}) \geq d-c+1$, then $X$ is of maximal sectional regularity and $\mathbb{F}=\mathbb{F}(X)$.

(b) Suppose that $X$ is a variety of maximal sectional regularity. If $\mathbb{L}_{\Lambda} \subset \mathbb{F}$ for general $\Lambda \in \mathcal{U}(X)$, then $\mathbb{F}(X)=\mathbb{F}$.

Proof. (a): Set $t:=\operatorname{deg}_{\mathbb{F}}(X \cap \mathbb{F})$ and let $\Lambda=\mathbb{P}^{c+1} \in \mathbb{G}\left(c+1, \mathbb{P}^{r}\right)$ be a general member. Then the line $\mathbb{L}:=\mathbb{F} \cap \Lambda$ is $t$-secant to the integral curve $\mathcal{C}_{\Lambda}:=X \cap \Lambda \subset \mathbb{P}^{c+1}$ of degree $d$. Therefore $t \leq \operatorname{reg}\left(\mathcal{C}_{\Lambda}\right) \leq d-c+1$, whence $t=d-c+1$. Thus $\mathcal{C}_{\Lambda} \subset \mathbb{P}^{c+1}$ is a curve of maximal regularity and $\mathbb{L} \subset \mathbb{F}$ is its unique $(d-c+1)$-secant line. This shows that $X$ is a variety of maximal sectional regularity and $\mathbb{F} \subseteq \mathbb{F}(X)$. Now, let $\Lambda \in \mathcal{U}(X)$ and consider the integral curve $\mathcal{C}_{\Lambda}:=X \cap \Lambda \subset \mathbb{P}^{c+1}$ of maximal regularity. It is clear that $\mathbb{L}:=\mathbb{F} \cap \Lambda$ is a line such that length $\left(\mathcal{C}_{\Lambda} \cap \mathbb{L}\right) \geq \mathrm{d}-\mathrm{c}+1$. Thus $\mathbb{L}$ is the unique $(d-c+1)$-secant line to $\mathcal{C}_{\Lambda}$. This shows that $\mathbb{F}=\mathbb{F}(X)$.

(b): For general $\Lambda \in \mathcal{U}(X)$, we have $\mathbb{L}_{\Lambda}:=\mathbb{F} \cap \Lambda$ and thus

$$
d-c+1=\operatorname{length}\left(\mathcal{C}_{\Lambda} \cap \mathbb{L}_{\Lambda}\right) \leq \operatorname{length}\left(\mathrm{X} \cap \mathbb{L}_{\Lambda}\right)=\operatorname{length}\left((\mathrm{X} \cap \mathbb{F}) \cap \mathbb{L}_{\Lambda}\right)=\operatorname{deg}_{\mathbb{F}}(\mathrm{X} \cap \mathbb{F}) .
$$

So, our claim follows by statement (a).

5.2. Proposition. Let $X \subset \mathbb{P}^{r}$ be a nondegenerate irreducible projective variety of dimension $n$, codimension $c \geq 3$ and degree $d \geq c+3$. If $X$ is a variety of maximal sectional regularity, then the following conditions are equivalent.

(i) $\mathbb{F}(X)$ is an $n$-dimensional linear space.

(ii) $\operatorname{dim} \mathbb{F}(X)=n$.

Proof. (i) $\Rightarrow$ (ii) is obvious.

(ii) $\Rightarrow$ (i): Let $D_{1}, \ldots, D_{t}$ be the different $n$-dimensional irreducible components of $\mathbb{F}(X)$ and write

$$
\mathbb{F}(X)=D_{1} \cup \cdots \cup D_{t} \cup E
$$

where $E \subset \mathbb{P}^{r}$ is a closed subset of dimension at most $n-1$. Also we write $X \cap D_{j}$ as $V_{j} \cup W_{j}$ where $V_{j}$ is either empty of else an equidimensional scheme of dimension $n-1$ and $W_{j}$ is a closed subscheme of dimension at most $n-2$. Now, choose a general $\Lambda \in \mathcal{U}(X)$. So, it avoids $W_{1}, \ldots, W_{t}$ and $E \cap \Lambda$ is at most a finite set. Then we have

$$
\mathbb{L}_{\Lambda} \subseteq \mathbb{F}(X) \cap \Lambda=\left(D_{1} \cap \Lambda\right) \cup \cdots \cup\left(D_{t} \cap \Lambda\right) \cup(E \cap \Lambda)
$$

and hence $\mathbb{L}_{\Lambda}=D_{i} \cap \Lambda$ for some $i$. This means that $D_{i}$ is a linear space. Also

$$
\mathcal{C}_{\Lambda} \cap \mathbb{L}_{\Lambda}=\left(X \cap D_{i}\right) \cap \Lambda=V_{i} \cap \mathbb{L}_{\Lambda}
$$

is nonempty and hence $X \cap D_{j}$ is of dimension $n-1$. Furthermore, we have

$$
\operatorname{deg}_{D_{i}}\left(X \cap D_{i}\right)=\operatorname{deg}_{D_{i}} V_{i}=\operatorname{length}\left(X \cap D_{i} \cap \Lambda^{\prime}\right)=\operatorname{length}\left(V_{i} \cap \mathbb{L}_{\Lambda}\right)=d-c+1 .
$$

Therefore it follows by Lemma 5.1 (a) that $\mathbb{F}(X)$ coincides with $D_{i}$. 
5.3. Lemma. Let $\widetilde{X}=S(\underbrace{0, \ldots, 0}_{k-\text { times }}, a_{k+1}, \ldots, a_{n}) \subset \mathbb{P}^{d+n-1}$ be a rational normal $n$-fold scroll for some $0 \leq k \leq n-2$ and positive integers $a_{k+1} \leq \ldots \leq a_{n}$. Let $D \subset \tilde{X}$ be a divisor linearly equivalent to $s H+t F$ where $H$ is a hyperplane section of $\widetilde{X}$ and $F \subset \widetilde{X}$ is an $(n-1)$-dimensional linear space. Then

(a) If $s \geq 2$ or $s=1$ and $t>0$ or $s=0$ and $t>a_{n}$, then $\langle D\rangle=\mathbb{P}^{d+n-1}$.

(b) If $s=1$ and $t \leq 0$, then $\operatorname{dim}\langle\mathrm{D}\rangle=\mathrm{d}+\mathrm{n}+\mathrm{t}-2$.

(c) If $s=0$ and $a_{i-1}<t \leq a_{i}$ for some $i \leq n$, then

$$
\operatorname{dim}\langle\mathrm{D}\rangle=\left(\mathrm{a}_{\mathrm{k}+1}+\cdots+\mathrm{a}_{\mathrm{i}-1}\right)+(\mathrm{n}-\mathrm{i}+1) \mathrm{t}+(\mathrm{i}-2) .
$$

(d) If $s=1$ and $t \leq 0$, then $I_{D}=I_{\widetilde{X}}+I_{\langle D\rangle}$.

Proof. First, observe that it is enough to show the statements in the case where $k=0$. So, we suppose that $\widetilde{X}$ is smooth. Let $R$ denote the homogeneous coordinate ring of $\mathbb{P}^{d+n-1}$. Also let $I_{D}$ and $\mathcal{I}_{D}$ be respectively the homogeneous ideal and the sheaf of ideals of $D$ in $\mathbb{P}^{d+n-1}$ and consider the exact sequence $0 \rightarrow \mathcal{I}_{\widetilde{X}} \rightarrow \mathcal{I}_{D} \rightarrow \mathcal{O}_{\widetilde{X}}(-D) \rightarrow 0$. Since $\tilde{X}$ is a projectively normal variety, we get the exact sequence

$$
0 \rightarrow I_{\widetilde{X}} \rightarrow I_{D} \rightarrow E=\bigoplus_{j \in \mathbb{Z}} E_{j} \rightarrow 0
$$

of graded $R$-modules where $E_{j}:=H^{0}\left(\widetilde{X}, \mathcal{O}_{\widetilde{X}}(j H-D)\right)$.

(a) (c): From the above short exact sequence (5.1), we know that

$$
\operatorname{dim}\langle\mathrm{D}\rangle=\mathrm{d}+\mathrm{n}-1-\mathrm{h}^{0}\left(\widetilde{\mathrm{X}}, \mathcal{O}_{\widetilde{\mathrm{X}}}(\mathrm{H}-\mathrm{D})\right) .
$$

If $s \geq 2$, then $H^{0}\left(\widetilde{X}, \mathcal{O}_{\widetilde{X}}(H-D)\right)=0$ and hence $D$ spans the whole ambient space. If $s=1$, then $H^{0}\left(\widetilde{X}, \mathcal{O}_{\widetilde{X}}(H-D)\right) \cong H^{0}\left(\mathbb{P}^{1}, \mathcal{O}_{\mathbb{P}^{1}}(-t)\right)$ and hence we get the desired result. If $s=0$, then

$$
H^{0}\left(\widetilde{X}, \mathcal{O}_{\widetilde{X}}(H-D)\right) \cong \bigoplus_{k+1 \leq j \leq n} H^{0}\left(\mathbb{P}^{1}, \mathcal{O}_{\mathbb{P}^{1}}\left(a_{j}-t\right)\right)
$$

and hence again we get the desired formula.

(d): One can easily check that $\mathcal{O}_{\widetilde{X}}(H-D)$ is 1 -regular with respect to $\mathcal{O}_{\widetilde{X}}(H)$ and hence $E$ is generated by $E_{1}$. Then it follows from (5.1) that $I_{D}$ is generated by its degree one piece and $I_{\tilde{X}}$. This completes the proof.

5.4. Theorem. Suppose that $\operatorname{char}(\mathbb{k})=0$ or $n=2$ and let $X \subset \mathbb{P}^{r}$ be a nondegenerate irreducible projective variety of dimension $n \geq 2$, codimension $c \geq 3$ and degree $d \geq c+3$. Then the following two conditions are equivalent:

(i) $X$ is a variety of maximal sectional regularity and $\mathbb{F}(X)$ is an $n$-dimensional linear space.

(ii) Either $X$ is a cone over a curve of maximal regularity or else $X=\pi_{\Lambda}(\widetilde{X})$ where 1. $\tilde{X}=S(\underbrace{0, \ldots, 0}_{k-\text { times }}, a_{k+1}, \ldots, a_{n}) \subset \mathbb{P}^{d+n-1}$ is a rational normal $n$-fold scroll for some $0 \leq k \leq n-2$ and positive integers $a_{k+1} \leq \ldots \leq a_{n}$, 
2. $D \subset \widetilde{X}$ is an effective divisor linearly equivalent to $H+(1-c) F$ where $H$ is a hyperplane section of $\widetilde{X}$ and $F \subset \widetilde{X}$ is an $(n-1)$-dimensional linear space (hence $\left.\langle D\rangle=\mathbb{P}^{d-c+n-1}\right)$, and

3. $\Lambda \subset\langle D\rangle$ is a $(d-c-2)$-dimensional subspace such that the restriction $\pi_{\Lambda} \uparrow$ : $\langle D\rangle \backslash \Lambda \rightarrow \mathbb{P}^{n}$ is generically injective along $D$.

In this case, $X$ is singular.

Proof. (i) $\Rightarrow$ (ii) : Let $\widetilde{X} \subset \mathbb{P}^{d+n-1}$ and $\Lambda=\mathbb{P}^{d-c-2}$ be as in Corollary 4.3. Thus

$$
\tilde{X}=S(\underbrace{0, \ldots, 0}_{k-\text { times }}, a_{k+1}, \ldots, a_{n}) \subset \mathbb{P}^{d+n-1}
$$

for some $0 \leq k \leq n-1$ and positive integers $a_{k+1} \leq \ldots \leq a_{n}$. If $k=n-1$, then $X$ should be a cone over a curve of maximal regularity. Now, assume that $k<n-1$. Note that $G:=X \cap \mathbb{F}(X)$ contains a hypersurface of $\mathbb{F}(X)$ whose degree is at least $d-c+1$. Let $\mathbb{E}$ and $D$ be respectively the pre-images of $\mathbb{F}(X)$ and $G$ by $\pi_{\Lambda}$. Thus $\mathbb{E}$ is a $(d-c+n-1)$-dimensional linear space and $D \subset \mathbb{E}$ is a subscheme of dimension $n-1$ and degree $\geq d-c+1$. Thus $D$ contains a divisor $D^{\prime}$ of $\widetilde{X}$ whose degree is at least $d-c+1$. Let $s H+t F$ be the divisor class of $D^{\prime}$ in $\tilde{X}$. Thus we have the inequality $\operatorname{deg}\left(D^{\prime}\right)=s d+t \geq d-c+1$. Note that $\left\langle D^{\prime}\right\rangle$ is a subspace of $\mathbb{E}$ which is a proper subspace of $\mathbb{P}^{d+n-1}$. On the other hand, one can show by Lemma 5.3(a) (c) that if $s=0$ and hence $t \geq d-c+1$ or $s=1$ and $t>1-c$ or $s \geq 2$ then the dimension of $\left\langle D^{\prime}\right\rangle$ is strictly greater than that of $\mathbb{E}$. Therefore we have $s=1$ and $t=1-c$. Then it follows respectively by Lemma 5.3 (b) and (d) that

$$
(\dagger)\left\langle D^{\prime}\right\rangle=\mathbb{E} \text { and }(\ddagger) \tilde{X} \cap\left\langle D^{\prime}\right\rangle=D^{\prime}
$$

From $(\dagger)$, we know that $\langle D\rangle$ contains $\Lambda$. Also from $(\ddagger)$, it holds that $D=D^{\prime}$ and hence $G=\pi_{\Lambda}\left(D^{\prime}\right)$ is a hypersurface in $\mathbb{F}(X)$. Finally, the restriction $\pi_{\Lambda} \uparrow:\langle D\rangle \backslash \Lambda \rightarrow \mathbb{F}(X)$ is generically injective along $D$ since $D$ and $G$ have the same degree.

(i) $\Leftarrow($ ii $):$ Assume that $X$ is a cone over a curve $\mathcal{C} \subset \mathbb{P}^{c+1}$ of maximal regularity. Let $\Delta=\mathbb{P}^{n-2}$ be the vertex of $X$ and $\mathbb{L}_{\mathcal{C}}$ the unique $(d-c+1)$-secant line of $\mathcal{C}$. It is clear that $X$ is a variety of maximal sectional regularity. Also the $n$-dimensional linear space $\mathbb{F}:=\left\langle\mathbb{L}_{\mathcal{C}}, \Delta\right\rangle$ satisfies the conditions that $\operatorname{dim}(X \cap \mathbb{F})=n-1$ and $\mathbb{L}_{\Lambda^{\prime}} \subset \mathbb{F}$ for general $\Lambda^{\prime} \in \mathcal{U}(X)$. Therefore it follows by Lemma 5.1(b) that $\mathbb{F}(X)=\mathbb{F}$.

Now, consider the second case. Let $\mathbb{F}$ be the $n$-dimensional linear space $\pi_{\Lambda}(\mathbb{E} \backslash \Lambda)$. Note that $D$ is of degree $d-c+1$. As $\pi_{\Lambda} \uparrow:\langle D\rangle \backslash \Lambda \rightarrow \mathbb{F}$ is generically injective along $D$, the map $\pi_{\Lambda}: \tilde{X} \rightarrow X$ is birational and hence $G:=\pi_{\Lambda}(D) \subset \mathbb{F}$ is a codimension one subscheme of degree $d-c+1$. As $G \subset X \cap \mathbb{F}$ it holds that $X \cap \mathbb{F}$ is of dimension $n-1$ and of degree $\geq d-c+1$. It follows by Lemma 5.1 that $X$ is a variety of maximal sectional regularity and $\mathbb{F}(X)=\mathbb{F}$ is an $n$-dimensional linear space.

Finally, the map $\pi_{\Lambda} \uparrow: D \rightarrow G$ cannot be an isomorphism since $G$ in $\mathbb{P}^{n}$ is linearly normal. This implies that the finite birational morphism $\pi_{\Lambda}: \widetilde{X} \rightarrow X$ is not an isomorphism and hence $X$ is singular. 


\section{Surfaces of Maximal Sectional Regularity}

This section is aimed to classify projective surfaces of maximal sectional regularity. To this end, we will first classify the extremal varieties of surfaces of maximal sectional regularity. To give precise statements, we require some notation and definitions.

6.1. Notation and Remark. Let $r \geq 5$ and let $X \subset \mathbb{P}^{r}$ be a surface of degree $d \geq r+1$ and of maximal sectional regularity. Thus $\mathcal{U}(X)$ is a nonempty open subset of $\left(\mathbb{P}^{r}\right)^{*}$. For every $\mathbb{H} \in \mathcal{U}(X)$, the intersection $\mathcal{C}_{\mathbb{H}}:=X \cap \mathbb{H} \subset \mathbb{P}^{r-1}$ is an integral curve of maximal regularity. We denote by $\mathbb{L}_{\mathbb{H}}$ the unique $(d-r+3)$-secant line to $\mathcal{C}_{\mathbb{H}}$.

(A) Theorem 4.1 says that the normalization $\pi: \tilde{X} \rightarrow X$ of $X$ is realized as a linear projection of a rational normal surface scroll $\widetilde{X} \subset \mathbb{P}^{d+1}$. In particular, $X$ is covered by lines.

(B) By view of Theorem 4.1 it follows that the singular locus $\operatorname{Sing}(X)$ of $X$ is finite. Therefore the set

$$
\mathcal{V}(X):=\{\mathbb{H} \in \mathcal{U}(X) \mid \operatorname{Sing}(X) \cap \mathbb{H}=\emptyset\}
$$

is a nonempty open subset of $\mathcal{U}(X)$.

(C) For each $\mathbb{H} \in \mathcal{V}(X)$, consider the nondegenerate projective surface

$$
Z_{\mathbb{H}}:=\overline{\pi_{\mathbb{H}}\left(X \backslash \mathbb{L}_{\mathbb{H}}\right)} \subset \mathbb{P}^{r-2}
$$

where $\pi_{\mathbb{H}}: \mathbb{P}^{r} \backslash \mathbb{L}_{\mathbb{H}} \rightarrow \mathbb{P}^{r-2}$ is the linear projection from $\mathbb{L}_{\mathbb{H}}$. Since the intersection $X \cap \mathbb{L}_{\mathbb{H}}$ is contained in the smooth locus of $X$, we have

$$
\operatorname{deg}\left(Z_{\mathbb{H}}\right)=\operatorname{deg}(X)-\operatorname{length}\left(X \cap \mathbb{L}_{\mathbb{H}}\right)=d-(d-r+3)=r-3
$$

and hence $Z_{\mathbb{H}}$ is a surface of minimal degree. By $(\mathrm{A}), Z_{\mathbb{H}}$ is covered by lines and so it is a rational normal surface scroll. Write

$$
Z_{\mathbb{H}}=S\left(b_{\mathbb{H}}, r-3-b_{\mathbb{H}}\right)
$$

for some $0 \leq b_{\mathbb{H}} \leq \frac{r-3}{2}$. Whence the join

$$
W_{\mathbb{H}}:=\operatorname{Join}\left(\mathbb{L}_{\mathbb{H}}, X\right)=\operatorname{Join}\left(\mathbb{L}_{\mathbb{H}}, Z_{\mathbb{H}}\right)
$$

is a fourfold rational normal scroll $S\left(0,0, b_{\mathbb{H}}, r-3-b_{\mathbb{H}}\right)$ such that $S(0,0)=\mathbb{L}_{\mathbb{H}}$ and $S\left(b_{\mathbb{H}}, r-3-b_{\mathbb{H}}\right)=Z_{\mathbb{H}}$.

(D) Recall that $W_{\mathbb{H}}$ is cut out by quadrics. As $X$ is a subset of $W_{\mathbb{H}}$ and

$$
\operatorname{length}\left(\mathrm{X} \cap \mathbb{L}_{\mathbb{H}^{\prime}}\right)=\mathrm{d}-\mathrm{r}+3>2
$$

for any $\mathbb{H}^{\prime} \in \mathcal{U}(X)$, we have $\mathbb{F}(X) \subset W_{\mathbb{H}}$.

6.2. Proposition. Let $5 \leq r<d$ and let $X \subset \mathbb{P}^{r}$ be a surface of degree $d$ and of maximal sectional regularity. Let the notations be as in Notation and Remark 6.1.

(a) Suppose that $b_{\mathbb{H}}=0$ for some $\mathbb{H} \in \mathcal{V}(X)$. Then the following statements hold:

(1) $\mathbb{F}(X)$ is equal to the set of vertices of $W_{\mathbb{H}}$. In particular, it is a plane;

(2) For any $\mathbb{H}^{\prime} \in \mathcal{V}(X), W_{\mathbb{H}^{\prime}}=W_{\mathbb{H}}$ and hence $b_{\mathbb{H}^{\prime}}=0$.

(b) Suppose that $b_{\mathbb{H}}>0$ for some $\mathbb{H} \in \mathcal{V}(X)$. Then it holds

(1) $\mathbb{L}_{\mathbb{H}^{\prime}}$ is either equal or disjoint to $\mathbb{L}_{\mathbb{H}}$ for any $\mathbb{H}^{\prime} \in \mathcal{V}(X)$;

(2) $\operatorname{dim} \mathbb{F}(X)=3$ and $X \subset \mathbb{F}(X)$; 
(3) $r=5$;

(4) $b_{\mathbb{H}^{\prime}}=1$ for all $\mathbb{H}^{\prime} \in \mathcal{V}(X)$.

Proof. (a) Let $\mathbb{F}$ be the plane $S(0,0,0)$ of vertices of $W_{\mathbb{H}}$. Thus we have $W_{\mathbb{H}}=\operatorname{Join}(\mathbb{F}, X)$. For a general member $\mathbb{H}^{\prime} \in \mathcal{V}(X)$, we have

$$
\mathcal{C}_{\mathbb{H}^{\prime}} \subset W_{\mathbb{H}} \cap \mathbb{H}^{\prime}=S(0,0, r-3)
$$

where the line $S(0,0)$ in $W_{\mathbb{H}} \cap \mathbb{H}^{\prime}$ is equal to the intersection $\mathbb{F} \cap \mathbb{H}^{\prime}$. By Proposition 2.3, this line is the $(d-r+3)$-secant line $\mathbb{L}_{\mathbb{H}^{\prime}}$ of $\mathcal{C}_{\mathbb{H}^{\prime}}$ and hence $\mathbb{L}_{\mathbb{H}^{\prime}}$ is contained in $\mathbb{F}$. Furthermore, $X \cap \mathbb{F}$ is of dimension one. Thus, $\mathbb{F}(X)$ is exactly equal to the plane $\mathbb{F}$ by Lemma 5.1. Moreover the above (6.1) holds for all $\mathbb{H}^{\prime} \in \mathcal{V}(X)$. This implies that

$$
W_{\mathbb{H}^{\prime}}=\operatorname{Join}\left(\mathbb{L}_{\mathbb{H}^{\prime}}, X\right) \subset \operatorname{Join}(\mathbb{F}, X)=W_{\mathbb{H}} .
$$

Therefore we have $W_{\mathbb{H}^{\prime}}=W_{\mathbb{H}}$ and $b_{\mathbb{H}^{\prime}}=0$.

(b) Suppose that $b_{\mathbb{H}}>0$ for some $\mathbb{H} \in \mathcal{V}(X)$. Let $f: X \backslash \mathbb{L}_{\mathbb{H}} \rightarrow Z_{\mathbb{H}}$ be the restriction of the linear projection map from $\mathbb{L}_{\mathbb{H}}$. By Notation and Remark 6.1. (A) and (C), we know that $f$ is a birational map, $X$ is covered by lines and those lines map to the ruling lines of the smooth rational normal surface scroll $Z_{\mathbb{H}}$.

Let $\mathbb{H}^{\prime} \in \mathcal{V}(X)$. Then we claim that the lines $\mathbb{L}_{\mathbb{H}^{\prime}}$ and $\mathbb{L}_{\mathbb{H}}$ are either disjoint or else $\mathbb{L}_{\mathbb{H}^{\prime}}=\mathbb{L}_{\mathbb{H}}$. Consider the curve $\mathcal{C}^{\prime}:=\overline{f\left(\mathcal{C}_{\mathbb{H}^{\prime}} \backslash \mathbb{L}_{\mathbb{H}}\right)}$. The map $f \uparrow: \mathcal{C}_{\mathbb{H}^{\prime}} \backslash \mathbb{L}_{\mathbb{H}} \rightarrow \mathcal{C}^{\prime}$ extends to a unique birational morphism $g: \mathcal{C}_{\mathbb{H}^{\prime}} \rightarrow \mathcal{C}^{\prime}$ since $\mathcal{C}_{\mathbb{H}^{\prime}}$ is smooth. Also $\mathcal{C}^{\prime}$ and a general ruling line of $Z_{\mathbb{H}}$ intersect at a point. This means that $\mathcal{C}^{\prime}$ is a section of $Z_{\mathbb{H}}$ and hence it is a smooth rational curve. So, the morphism $g$ is indeed an isomorphism. Assume that $\mathbb{L}_{\mathbb{H}}$ and $\mathbb{L}_{\mathbb{H}^{\prime}}$ are different and not disjoint. Then they must meet at the vertex $q$ of the threefold scroll $W_{\mathbb{H}} \cap \mathbb{H}^{\prime}=S\left(0, b_{\mathbb{H}}, r-3-b_{\mathbb{H}}\right)$. Hence, the point $p=\pi_{\mathbb{H}}\left(\mathbb{L}_{\mathbb{H}} \backslash\{q\}\right) \in Z_{\mathbb{H}}$ satisfies

$$
p \in C^{\prime} \quad \text { and } \quad \operatorname{length}\left(\mathrm{g}^{-1}(\mathrm{p})\right)=\operatorname{length}\left(\mathbb{L}_{\mathbb{H}^{\prime}} \cap \mathrm{C}_{\mathbb{H}^{\prime}}\right)=\mathrm{d}-\mathrm{r}+3>1,
$$

which is a contradiction. This proves the stated disjointness of $\mathbb{L}_{\mathbb{H}^{\prime}}$ and $\mathbb{L}_{\mathbb{H}}$.

The previous disjointness implies that $\mathbb{F}(X)$ cannot be a plane since it should contain two disjoint lines. It follows by Proposition 5.2 that the dimension of $\mathbb{F}(X)$ is at least 3 . On the other hand, we will show that $\mathbb{F}(X)$ has dimension at most 3 . To do so, let us consider the incidence correspondence

$$
\Sigma:=\left\{(x, \mathbb{L}) \in \mathbb{P}^{r} \times \mathbb{G}\left(1, \mathbb{P}^{r}\right) \mid x \in \mathbb{L}\right\} \subseteq \mathbb{P}^{r} \times \mathbb{G}\left(1, \mathbb{P}^{r}\right)
$$

and the canonical projections

$$
\varphi: \Sigma \rightarrow \mathbb{P}^{r} \text { and } \psi: \Sigma \rightarrow \mathbb{G}\left(1, \mathbb{P}^{r}\right) .
$$

Theorem 3.4 says that $\overline{\sum_{d-c+1}^{o}(X)}$ is 2-dimensional and so $\psi^{-1}\left(\overline{\sum_{d-c+1}^{o}(X)}\right)$ is 3-dimensional. Therefore the closed subset

$$
\varphi\left(\psi^{-1}\left(\overline{\sum_{d-c+1}^{o}(X)}\right)\right)=\bigcup_{\mathbb{L} \in \overline{\Sigma_{d-c+1}^{o}(X)}} \mathbb{L} \subset \mathbb{P}^{r}
$$

has dimension $\leq 3$. Since $\mathbb{F}(X)$ is contained in this closed subset, we have $\operatorname{dim} \mathbb{F}(X) \leq 3$. In consequence, it is shown that

$$
\operatorname{dim} \mathbb{F}(X)=\operatorname{dim} \varphi\left(\psi^{-1}\left(\overline{\sum_{d-c+1}^{o}(X)}\right)\right)=3 .
$$


Now, we will show that $X$ is a subset of $\mathbb{F}(X)$. To this aim, consider the set

$$
\Xi(X):=\left\{\mathbb{L}_{\mathbb{H}} \mid \mathbb{H} \in \mathcal{V}(X)\right\} .
$$

From the the proof of Proposition 3.3 one can see that $\Xi(X)$ is 2-dimensional and $\Sigma_{d-c+1}^{o}(X) \backslash \Xi(X)$ is of dimension at most one. This means that the coincidence set

$$
Y:=\psi^{-1}(\Xi(X))=\left\{\left(x, \mathbb{L}_{\mathbb{H}}\right) \mid \mathbb{H} \in \mathcal{V}(X) \text { and } x \in \mathbb{L}_{\mathbb{H}}\right\}
$$

is 3-dimensional and its subset

$$
T:=Y \cap\left(X \times \mathbb{G}\left(1, \mathbb{P}^{r}\right)\right)=\left\{\left(x, \mathbb{L}_{\mathbb{H}}\right) \mid \mathbb{H} \in \mathcal{U}(X) \text { and } x \in X \cap \mathbb{L}_{\mathbb{H}}\right\}
$$

is 2-dimensional. Now, the previous disjointness implies that the projection map

$$
\varphi \uparrow: T \rightarrow X \cap \mathbb{F}(X)
$$

is injective. It follows that $X \cap \mathbb{F}(X)$ is 2-dimensional and so $X \subset \mathbb{F}(X)$.

Next, we will prove that $b_{\mathbb{H}}=1$. Recall that $\mathbb{L}_{\mathbb{H}^{\prime}}$ and $\mathbb{L}_{\mathbb{H}}$ are disjoint if $\mathbb{L}_{\mathbb{H}^{\prime}} \neq \mathbb{L}_{\mathbb{H}}$. This implies that the line $\mathbb{L}_{\mathbb{H}^{\prime}}$ avoids the vertex $q$ of the threefold rational normal scroll $W_{\mathbb{H}} \cap \mathbb{H}^{\prime}=S\left(0, b_{\mathbb{H}}, r-3-b_{\mathbb{H}}\right)$ and that it is not contained in any of the ruling planes of $W_{\mathbb{H}} \cap \mathbb{H}^{\prime}$. Therefore $\mathbb{M}_{\mathbb{H}^{\prime}}:=\pi_{\mathbb{L}_{\mathbb{H}}}\left(\mathbb{L}_{\mathbb{H}^{\prime}}\right)$ is a line such that $g\left(C_{\mathbb{H}^{\prime}} \cap \mathbb{L}_{\mathbb{H}^{\prime}}\right) \subset Z_{\mathbb{H}} \cap \mathbb{M}_{\mathbb{H}^{\prime}}$. As $g$ is an isomorphism, we have

$$
\text { length }\left(\mathrm{C}^{\prime} \cap \mathbb{M}_{\mathbb{H}^{\prime}}\right)=\text { length }\left(\mathrm{C}_{\mathbb{H}^{\prime}} \cap \mathbb{L}_{\mathbb{H}^{\prime}}\right)=\mathrm{d}-\mathrm{r}+3>2 \text {. }
$$

Then $\mathbb{M}_{\mathbb{H}^{\prime}} \subset Z_{\mathbb{H}}$ since $Z_{\mathbb{H}}$ is cut out by quadrics (cf. Notation and Remarks $2.2(\mathrm{~A})$ ). Furthermore, $\mathbb{M}_{\mathbb{H}}$ is not a ruling line of $Z_{\mathbb{H}}$ since the ruling lines of $Z_{\mathbb{H}}$ are precisely the images of the ruling planes of $W_{\mathbb{H}} \cap \mathbb{H}^{\prime}$ by the linear projection map from $q$. Consequently, $\mathbb{M}_{\mathbb{H} \mathbb{H}^{\prime}}$ must be a line section of $Z_{\mathbb{H}}$. In particular, $b_{\mathbb{H}}=1$.

Now, we will show that $r=5$. Assume to the contrary, that $r \geq 6$. Note that $\mathbb{L}_{\mathbb{H}^{\prime}}$ is contained in $W_{\mathbb{H}} \cap \mathbb{H}^{\prime}=S(0,1, r-4)$ while it is not contained in any of the ruling planes of $W_{\mathbb{H}} \cap \mathbb{H}^{\prime}$. This means that $\mathbb{L}_{\mathbb{H}}$ is contained in the plane $S(0,1)$ of $W_{\mathbb{H}} \cap \mathbb{H}^{\prime}$. It follows that the 3 -space $S(0,0,1)$ of $W_{\mathbb{H}}$ contains $\mathbb{L}_{\mathbb{H}^{\prime}}$ for all $\mathbb{H}^{\prime} \in \mathcal{V}(X)$ and hence $\mathbb{F}(X) \subset S(0,0,1)$. This is impossible since $\mathbb{F}(X)$ contains $X$ and hence it spans $\mathbb{P}^{r}$. This contradiction shows that indeed $r=5$.

Finally, note that since $b_{\mathbb{H}}>0$ we have $b_{\mathbb{H}^{\prime}}>0$ for all $\mathbb{H}^{\prime} \in \mathcal{V}(X)$ by $(a)$. Then applying the previous arguments to $\mathbb{H}^{\prime}$, we get the desired equality $b_{\mathbb{H}^{\prime}}=1$.

Now, we are ready to prove the following complete classification result of surfaces of maximal sectional regularity.

6.3. Theorem. Let $5 \leq r<d$ and let $X \subset \mathbb{P}^{r}$ be a nondegenerate irreducible projective surface of degree $d \geq r+1$. If $X$ is a surface of maximal sectional regularity, then either $\mathbb{F}(X)$ is a plane or else $r=5, \mathbb{F}(X)=S(1,1,1)$ and $X \subset \mathbb{F}(X)$. Moreover we have

(a) The following two statements are equivalent:

(i) $X$ is a surface of maximal sectional regularity and $\mathbb{F}(X)$ is a plane.

(ii) $X$ is either a cone over a curve of maximal regularity or else a projection $\pi_{\Lambda}(\widetilde{X})$ of a smooth rational normal surface scroll $\widetilde{X} \subset \mathbb{P}^{d+1}$ where

1. $D \subset \widetilde{X}$ is an effective divisor linearly equivalent to $H+(1-c) F$ where $H$ and $F$ are respectively a hyperplane divisor and a ruling line of $\widetilde{X}$ (hence $\langle D\rangle=\mathbb{P}^{d-r+3}$ ), and 
2. $\Lambda \subset\langle D\rangle$ is a $(d-r)$-dimensional subspace such that the restriction $\pi_{\Lambda} \uparrow:\langle D\rangle \backslash \Lambda \rightarrow \mathbb{P}^{2}$ is generically injective along $D$.

In this case, $X$ is singular.

(b) The following two statements are equivalent:

(i) $X$ is a surface of maximal sectional regularity and $\mathbb{F}(X)=S(1,1,1)$.

(ii) $X$ is contained in $S(1,1,1)$ as a divisor linearly equivalent to $H+(d-3) F$, where $H$ is the hyperplane divisor and $F$ is a ruling plane of $S(1,1,1)$.

In this case, $X$ is smooth.

Proof. Suppose that $\mathbb{F}(X)$ is not a plane. Then Proposition 6.2 shows that $r=5$, $\operatorname{dim} \mathbb{F}(X)=3$ and $X \subset \mathbb{F}(X)$. Thus it remains to prove that $\mathbb{F}(X)=S(1,1,1)$. To this aim, let $\mathbb{H}, \mathbb{H}^{\prime} \in \mathcal{V}(X)$ such that $\mathbb{L}_{\mathbb{H}} \neq \mathbb{L}_{\mathbb{H}}^{\prime}$. Then Proposition 6.2(b) says that the two quadrics $W_{\mathbb{H}}$ and $W_{\mathbb{H}^{\prime}}$ in $\mathbb{P}^{5}$ are both of type $S(0,0,1,1)$ and $\left\langle\mathbb{L}_{\mathbb{H}}, \mathbb{L}_{\mathbb{H}^{\prime}}\right\rangle$ is a 3 -space. Moreover, as $\mathbb{L}_{\mathbb{H}}$ is the vertex of $W_{\mathbb{H}}=S(0,0,1,1)$ and $\mathbb{L}_{\mathbb{H}^{\prime}} \subset W_{\mathbb{H}}$ it holds $\left\langle\mathbb{L}_{\mathbb{H}}, \mathbb{L}_{\mathbb{H}^{\prime}}\right\rangle \subset W_{\mathbb{H}}$. Hence, by symmetry we get

$$
X \subset \mathbb{F}(X) \subset W_{\mathbb{H}} \cap W_{\mathbb{H}^{\prime}} \quad \text { and } \quad \mathbb{P}^{3}=\left\langle\mathbb{L}_{\mathbb{H}}, \mathbb{L}_{\mathbb{H}}\right\rangle \subset W_{\mathbb{H}} \cap W_{\mathbb{H}^{\prime}}
$$

As $W_{\mathbb{H}}$ and $W_{\mathbb{H}^{\prime}}$ are two distinct integral hyperquadrics in $\mathbb{P}^{5}$, we know that $W_{\mathbb{H}^{H}} \cap W_{\mathbb{H}^{\prime}}$ is a complete intersection and hence a 3-dimensional arithmetically Cohen-Macaulay scheme of degree 4. As $X \subset \mathbb{P}^{5}$ is non-degenerate and contained in $W_{\mathbb{H}} \cap W_{\mathbb{H}^{\prime}}$, it follows that

$$
W_{\mathbb{H}} \cap W_{\mathbb{H}^{\prime}}=\left\langle\mathbb{L}_{\mathbb{H}}, \mathbb{L}_{\mathbb{H}^{\prime}}\right\rangle \cup V
$$

for a 3-dimensional non-degenerate integral closed subscheme $V \subset \mathbb{P}^{5}$ of degree 3 . Note that $V$ is a scroll of type $S(1,1,1)$ or $S(0,1,2)$ or $S(0,0,3)$. In particular, $V$ is cut out by quadrics. Also $X \subset V$. Therefore $\mathbb{L}_{\mathbb{H}^{\prime \prime}} \subset V$ for all $\mathbb{H}^{\prime \prime} \in \mathcal{V}(X)$ and hence that $\mathbb{F}(X)=V$. Finally, we aim to exclude the latter two cases. First, $V$ cannot be equal to $S(0,0,3)$ since any divisor of $S(0,0,3)$ is arithmetically Cohen-Macaulay while $X$ is not. Now, assume that $V=S(0,1,2)$ and let $\mathbb{H}^{\prime \prime} \in \mathcal{V}(X)$ be a general member. Then, we have

$$
C_{\mathbb{H}^{\prime \prime}} \subset V \cap \mathbb{H}^{\prime \prime}=S(1,2) \subset \mathbb{H}^{\prime \prime}=\mathbb{P}^{4},
$$

and according to Proposition 2.4 . (c), the line section $S(1)$ of $S(1,2)$ coincides with $\mathbb{L}_{\mathbb{H}^{\prime \prime}}$. Therefore $\mathbb{L}_{\mathbb{H}^{\prime \prime}}$ should lie on the plane $S(0,1)$ and hence this plane is $\mathbb{F}(X)$, which is a contradiction. Therefore $\mathbb{F}(X)=V$ is equal to $S(1,1,1)$.

(a) This follows immediately from Theorem 5.4 .

(b) (i) $\Longrightarrow$ (ii) : Note that $X$ is contained in $S(1,1,1)$ since $\mathbb{F}(X)$ is not a plane. For all $\mathbb{H} \in \mathcal{U}(X)$, we have

$$
C_{\mathbb{H}}=X \cap \mathbb{H} \subset \mathbb{F}(X) \cap \mathbb{H}=S(1,2) \subset \mathbb{P}^{4}
$$

and Proposition 2.4. (b) yields that the divisor $X$ is linearly equivalent to $H+(d-3) F$.

(i) $\Longleftarrow$ (ii) : It is clear that any line in the 2-dimensional family of line sections of $S(1,1,1)$ is a $(d-r+3)$-secant line to $X$. Then Theorem 3.4. (b) shows that $\overline{\mathfrak{d}}_{d-r+3}(X)$ is equal to 2 and so $X$ is a surface of maximal sectional regularity. Furthermore, $S(1,1,1)$ is contained in $\mathbb{F}(X)$ and hence we get the desired equality $S(1,1,1)=\mathbb{F}(X)$ by our previous classification result of $\mathbb{F}(X)$.

It remains to show that $X$ is smooth. One can easily check that the $\Delta$-genus of $\left(X, \mathcal{O}_{X}(1)\right)$ is equal to zero. This implies that the linearly normal embedding of $X$ by 
$\mathcal{O}_{X}(1)$, say $\tilde{X} \subset \mathbb{P}^{d+1}$, is a rational normal surface scroll and $X$ is the image of an isomorphic linear projection of $\widetilde{X}$. Since $\widetilde{X}$ admits an isomorphic linear projection, it is not a cone and hence a smooth rational normal surface scroll. Therefore $X$ is a smooth surface.

6.4. Remark. Let $X$ be as in Theorem 6.3(b)(ii). Thus it is contained in $Y:=S(1,1,1)$ as a divisor linearly equivalent to $H+(d-3) F$, where $H$ is the hyperplane divisor and $F$ is a ruling plane of $S(1,1,1)$. One can easily check that $H^{0}\left(Y, \mathcal{O}_{Y}(2 H-X)\right)=0$ (cf. Notation and Remarks $2.2(\mathrm{~B}))$. From the exact sequence

$$
0 \rightarrow \mathcal{I}_{Y} \rightarrow \mathcal{I}_{X} \rightarrow \mathcal{O}_{Y}(-X) \rightarrow 0
$$

it follows that $H^{0}\left(\mathbb{P}^{5}, \mathcal{I}_{Y}(2)\right)=H^{0}\left(\mathbb{P}^{5}, \mathcal{I}_{X}(2)\right)$. In particular, $I_{X}$ requires exactly three quadratic generators.

\section{Classification of varieties of maximal Sectional Regularity}

This section is aimed to prove the following classification of varieties of maximal sectional regularity in the case where the dimension and the codimension are both at least three.

7.1. Theorem. Suppose that char $(\mathbb{k})=0$ and let $X \subset \mathbb{P}^{r}$ be a nondegenerate irreducible projective variety of dimension $n \geq 3$, codimension $c \geq 3$ and degree $d \geq c+3$. If $X$ is a variety of maximal sectional regularity, then either $\mathbb{F}(X)$ is an n-dimensional linear space or else $c=3$ and $\mathbb{F}(X)=S(\underbrace{0, \ldots, 0}_{(n-2) \text {-times }}, 1,1,1)$. In the first case, $X \cap \mathbb{F}(X)$ in $\mathbb{F}(X)$ is a hypersurface of degree $d-c+1$. In the second case, $\mathbb{F}(X)$ contains $X$. Moreover we have

(a) The following two statements are equivalent:

(i) $X$ is a variety of maximal sectional regularity and $\mathbb{F}(X)$ is an $n$-dimensional linear space.

(ii) Either $X$ is a cone over a curve of maximal regularity or else $X=\pi_{\Lambda}(\tilde{X})$ where

1. $\widetilde{X}=S(\underbrace{0, \ldots, 0}_{k-\text { times }}, a_{k+1}, \ldots, a_{n}) \subset \mathbb{P}^{d+n-1}$ is a rational normal $n$-fold scroll for some $0 \leq k \leq n-2$ and positive integers $a_{k+1} \leq \ldots \leq a_{n}$,

2. $D \subset \widetilde{X}$ is an effective divisor linearly equivalent to $H+(1-c) F$ where $H$ is a hyperplane divisor of $\widetilde{X}$ and $F \subset \widetilde{X}$ is an $(n-1)$-dimensional linear space (hence $\langle D\rangle=\mathbb{P}^{d-r+2 n-1}$ ), and

3. $\Lambda \subset\langle D\rangle$ is a $(d-c-2)$-dimensional subspace such that the restriction $\pi_{\Lambda} \uparrow:\langle D\rangle \backslash \Lambda \rightarrow \mathbb{P}^{n}$ is generically injective along $D$.

(b) The following two statements are equivalent:

(i) $X$ is a variety of maximal sectional regularity and $\mathbb{F}(X)=S(\underbrace{0, \ldots, 0}_{(n-2) \text {-times }}, 1,1,1)$.

(ii) $X$ is contained in the $(n+1)$-fold scroll $Y:=S(\underbrace{0, \ldots, 0}_{(n-2) \text {-times }}, 1,1,1)$ as a divisor linearly equivalent to $H+(d-3) F$, where $H$ is the hyperplane divisor of $Y$ and $F \subset Y$ is an $n$-dimensional linear space. 
To prove this theorem, we need the following two lemmas.

7.2. Lemma. Let $X \subset \mathbb{P}^{r}$ be a variety of maximal sectional regularity of dimension $n \geq 2$ and codimension $c \geq 3$. If $\mathbb{F}(S)$ is a plane for a general linear surface section $S \subset \mathbb{P}^{r-n+2}$ of $X$, then $\mathbb{F}(X)$ is an $n$-dimensional linear space.

Proof. We use induction on $n \geq 2$.

The statement is obvious for $n=2$.

Suppose that $n \geq 3$. By induction hypothesis, if $\mathbb{H}_{1}$ and $\mathbb{H}_{2}$ are general hyperplanes of $\mathbb{P}^{r}$ then $\Lambda_{1}:=\mathbb{F}\left(X \cap \mathbb{H}_{1}\right)$ and $\Lambda_{2}:=\mathbb{F}\left(X \cap \mathbb{H}_{2}\right)$ are $(n-1)$-dimensional linear spaces. Furthermore, $\mathbb{F}\left(X \cap \mathbb{H}_{1} \cap \mathbb{H}_{2}\right)$ is an $(n-2)$-dimensional linear subspace (either by Notation and Remarks 2.1(A) for $n=3$ and by induction if $n>3$ ), which is contained in $\Lambda_{1} \cap \Lambda_{2}$. Thus the linear space

$$
\Lambda:=\left\langle\Lambda_{1}, \Lambda_{2}\right\rangle
$$

is of dimension $n$. Now, let $\mathbb{H}$ be a general hyperplane. Then $\mathbb{F}(X \cap \mathbb{H})$ is an $(n-1)$ dimensional linear space. Also we know that $\mathbb{F}\left(X \cap \mathbb{H} \cap \mathbb{H}_{1}\right)$ and $\mathbb{F}\left(X \cap \mathbb{H} \cap \mathbb{H}_{2}\right)$ are $(n-2)$-dimensional linear subspaces (again, either by Notation and Remarks 2.1(A) for $n=3$ or by induction if $n>3$ ) and hence that

$$
\mathbb{F}(X \cap \mathbb{H})=\left\langle\mathbb{F}\left(X \cap \mathbb{H} \cap \mathbb{H}_{1}\right), \mathbb{F}\left(X \cap \mathbb{H} \cap \mathbb{H}_{2}\right)\right\rangle .
$$

Since $\mathbb{F}\left(X \cap \mathbb{H} \cap \mathbb{H}_{1}\right)$ and $\mathbb{F}\left(X \cap \mathbb{H} \cap \mathbb{H}_{2}\right)$ are respectively linear subspaces of $\Lambda_{1}$ and $\Lambda_{2}$, it follows that $\mathbb{F}(X \cap \mathbb{H})$ is contained in $\Lambda$. By Lemma 5.1, we conclude that $\mathbb{F}(X)$ is equal to the $n$-dimensional linear space $\Lambda$.

7.3. Lemma. Suppose that $\operatorname{char}(\mathbb{k})=0$ and let $X \subset \mathbb{P}^{6}$ be a 3-dimensional variety of maximal sectional regularity such that $\mathbb{F}(X \cap \mathbb{H})=S(1,1,1)$ for a hyperplane $\mathbb{H}=\mathbb{P}^{5}$. Then

(a) $\mathbb{F}(S)=S(1,1,1)$ for a general hyperplane section $S \subset \mathbb{P}^{5}$ of $X$.

(b) $\mathbb{F}(X)$ is the rational normal fourfold scroll $S(0,1,1,1)$ and $X$ is contained in $\mathbb{F}(X)$ as a divisor linearly equivalent to $H+(d-3) F$, where $H$ is a hyperplane divisor of $S(0,1,1,1)$ and $F$ is a linear 3 -space in $S(0,1,1,1)$.

Proof. (a) By Theorem 6.3(c) and Corollary 4.2, we have

$$
\chi\left(\mathcal{O}_{X \cap \mathbb{H}}(t)\right)=d\left(\begin{array}{c}
t+1 \\
2
\end{array}\right)+t+1 .
$$

Clearly, this implies that the Euler-Poincaré characteristic $\chi\left(\mathcal{O}_{X}(t)\right)$ is of the form

$$
\chi\left(\mathcal{O}_{X}(t)\right)=d\left(\begin{array}{c}
t+2 \\
3
\end{array}\right)+\left(\begin{array}{c}
t+1 \\
2
\end{array}\right)+t+\chi_{0}\left(\mathcal{O}_{X}(1)\right)
$$

and hence for a general linear surface section $S \subset \mathbb{P}^{5}$ of $X$, we have

$$
\chi\left(\mathcal{O}_{S}(t)\right)=d\left(\begin{array}{c}
t+1 \\
2
\end{array}\right)+t+1 .
$$

Thus $S$ is a smooth surface by Corollary 4.2. Now, from the classification result in Theorem 6.3 it follows that $\mathbb{F}(S)=S(1,1,1)$. 
(b) Recall that $X$ is a linear projection of a threefold rational normal scroll $\widetilde{X} \subset \mathbb{P}^{d+2}$ (cf. Corollary 4.3). If $\widetilde{X}$ is a cone over the rational normal curve, then $X$ should be a cone over a curve of maximal regularity and hence $\mathbb{F}(S)$ is a plane for a general hyperplane section $S \subset \mathbb{P}^{5}$ of $X$, a contradiction. Thus $\operatorname{Sing}(\widetilde{\mathrm{X}})$ is at most a point. By combining this with the fact that $\operatorname{dim} \operatorname{Sing}\left(\pi_{\Lambda}\right) \leq 1$ (cf. Theorem 4.1), we know that $\operatorname{dim} \operatorname{Sing}(\mathrm{X}) \leq 1$. Thus for general $\Lambda \in \mathcal{U}(X)$, the intersection $X \cap \mathbb{L}_{\Lambda}$ is contained in the smooth locus of $X$ and hence the join $Q_{\Lambda}:=\operatorname{Join}\left(\mathbb{L}_{\Lambda}, \mathrm{X}\right)$ is a quadratic hypersurface of $\mathbb{P}^{6}$ (cf. Notation and Remark 6.1). Now, choose two general members $\Lambda_{1}, \Lambda_{2} \in \mathcal{U}(X)$. Thus we have

$$
X \subset Q_{\Lambda_{1}} \cap Q_{\Lambda_{2}} \subset \mathbb{P}^{6}
$$

We will see that the intersection $Q_{\Lambda_{1}} \cap Q_{\Lambda_{2}}$ is reducible. Indeed, let $\mathbb{H}$ be a general hyperplane and $Q_{\Lambda_{i}, \mathbb{H}}=Q_{\Lambda_{i}} \cap \mathbb{H}(i=1,2)$. Then

$$
S:=X \cap \mathbb{H} \subset Q_{\Lambda_{1}, \mathbb{H}} \cap Q_{\Lambda_{2}, \mathbb{H}} \cdot
$$

Note that $I(S)_{2}=I(\mathbb{F}(S))_{2}$ (cf. Remark 6.4) and hence $Q_{\Lambda_{1}, \mathbb{H}}$ and $Q_{\Lambda_{2}, \mathbb{H}}$ are contained in $I(\mathbb{F}(S))_{2}$. Therefore the intersection $Q_{\Lambda_{1}, \mathbb{H}} \cap Q_{\Lambda_{2}, \mathbb{H}}$ is the union of the scroll $\mathbb{F}(S)=$ $S(1,1,1)$ and a linear subspace $\mathbb{P}^{3}$ of $\mathbb{H}$. Then, it is clear that $Q_{\Lambda_{1}} \cap Q_{\Lambda_{2}}$ should be the union of a scroll $Y:=S(0,1,1,1)$ and a 4-dimensional linear space. Obviously, our $X$ is contained in $Y$ as a divisor. Also from the divisor class of $S$ in $S(1,1,1)$, we know that $X$ is linearly equivalent to $H+(d-3) F$. Finally, $\mathbb{F}(X) \subset Y$ since $Y$ contains $X$ and it is cut out by quadrics. On the other hand, $Y \cap \mathbb{H}=\mathbb{F}(X \cap \mathbb{H}) \subset \mathbb{F}(X)$ for a general hyperplane $\mathbb{H}$ of $\mathbb{P}^{6}$. So, we get the desired equality $\mathbb{F}(X)=Y$.

Now we give the

Proof of Theorem 7.1. Let $S \subset \mathbb{P}^{c+2}$ be a general linear surface section of $X$. Then $S$ is a surface of maximal sectional regularity and either $\mathbb{F}(S)$ is a plane or else $c=3$, $\mathbb{F}(S)=S(1,1,1)$ and $S \subset \mathbb{F}(S)$ (cf. Theorem 6.3).

If $\mathbb{F}(S)$ is a plane for a general linear surface section $S \subset \mathbb{P}^{c+2}$, then $\mathbb{F}(X)$ is an $n$ dimensional linear space by Lemma 7.2 .

Now, consider the case where $c=3$ and there exists a 3-dimensional linear section $T \subset \mathbb{P}^{6}$ of $X$ which is a variety of maximal sectional regularity and which has an integral hyperplane section $S \subset \mathbb{P}^{5}$ of maximal sectional regularity such that $\mathbb{F}(S)=S(1,1,1)$. Then Lemma 7.3(b) shows that $\mathbb{F}(T)=S(0,1,1,1)$ and $T$ is contained in $\mathbb{F}(T)$ as a divisor linearly equivalent to $H+(d-3) F$, where $H$ is a hyperplane divisor of $S(0,1,1,1)$ and $F$ is a linear 3-space in $S(0,1,1,1)$. We first claim that for a general linear subspace $\mathbb{P}^{5} \subset \mathbb{P}^{n+3}$, the surface $X \cap \mathbb{P}^{5}$ satisfies the property that $\mathbb{F}\left(X \cap \mathbb{P}^{5}\right)=S(1,1,1)$. Indeed, by the same argument as in the proof of Lemma 7.3 (a), $S$ and $X \cap \mathbb{P}^{5}$ have the same Euler-Poincaré characteristic. Thus our claim is verified by Theorem 6.3. Now, observe that $\operatorname{depth}(\mathrm{T})$ is equal to 2 (cf. [19, Theorem 4.3]) and hence $\operatorname{depth}(\mathrm{X})=\mathrm{n}-1$. In particular, $I_{X}$ and $I_{S}$ require the same number of quadratic generators. Then it follows by Remark 6.4 that $I_{X}$ contains exactly three $\mathbb{k}$-linearly independent quadrics. Let $\left\{Q_{1}, Q_{2}, Q_{3}\right\}$ be a basis for $H^{0}\left(\mathbb{P}^{n+3}, \mathcal{I}_{X}(2)\right)$ and consider the closed subset $W \subset \mathbb{P}^{n+3}$ defined as the intersection of 
the three hyperquadrics $Q_{1}, Q_{2}$ and $Q_{3}$. We claim that

$$
W=S(\underbrace{0, \ldots, 0}_{(n-2) \text {-times }}, 1,1,1) .
$$

Indeed, for a general linear subspace $\mathbb{P}^{5} \subset \mathbb{P}^{n+3}$ consider the quadrics $Q_{i, \mathbb{P}^{5}}:=\left.Q_{i}\right|_{\mathbb{P}^{5}}$ $(i=1,2,3)$. Since $\operatorname{depth}(\mathrm{X})=\mathrm{n}-1$, we know that $\left\{Q_{1, \mathbb{P}^{5}}, Q_{2, \mathbb{P}^{5}}, Q_{3, \mathbb{P}^{5}}\right\}$ is a basis for $H^{0}\left(\mathbb{P}^{5}, \mathcal{I}_{X \cap \mathbb{P}^{5}}(2)\right)$. This implies that $W \cap \mathbb{P}^{5}$ which is the intersection of the quadrics $Q_{1, \mathbb{P}^{5}}, Q_{2, \mathbb{P}^{5}}$ and $Q_{3, \mathbb{P}^{5}}$ is precisely equal to the threefold scroll $\mathbb{F}\left(X \cap \mathbb{P}^{5}\right)$. Therefore $W$ contains a nondegenerate $(n+1)$-dimensional irreducible variety $W^{\prime}$ of degree 3 . Since $W^{\prime}$ is a variety of minimal degree and hence cut out by exactly three quadrics, we conclude that $W=W^{\prime}$. Also since $W \cap \mathbb{P}^{5}$ is equal to $S(1,1,1)$, it follows that $W$ is as above. Finally, note that $\mathbb{F}(X) \subset W$ since $W$ is cut out by quadrics. On the other hand, for a general linear subspace $\mathbb{P}^{5} \subset \mathbb{P}^{n+3}$ we have

$$
W \cap \mathbb{P}^{5}=\mathbb{F}\left(X \cap \mathbb{P}^{5}\right) \subset \mathbb{F}(X)
$$

which means that $W \subset \mathbb{F}(X)$. Therefore $W=\mathbb{F}(X)$.

(a) See Theorem 5.4.

(b) (i) $\Longrightarrow$ (ii) : Note that $X$ is contained in $\mathbb{F}(X)$ since $\mathbb{F}(X)$ is not a linear space. For general $\Lambda \in \mathcal{U}(X)$, we have

$$
\mathcal{C}_{\Lambda}=X \cap \Lambda \subset \mathbb{F}(X) \cap \Lambda=S(1,2) \subset \mathbb{P}^{4}
$$

and Proposition 2.4 (b) yields that the divisor $X$ is linearly equivalent to $H+(d-3) F$.

(i) $\Longleftarrow$ (ii) : Let $\mathbb{P}^{5} \in \mathbb{G}\left(5, \mathbb{P}^{n+3}\right)$ be a general member. Then we have

$$
S:=X \cap \mathbb{P}^{5} \subset Y \cap \mathbb{P}^{5}=S(1,1,1) \subset \mathbb{P}^{5}
$$

where $S$ is apparently a divisor of $S(1,1,1)$ linearly equivalent to $H_{0}+(d-3) F_{0}$, where $H_{0}$ is the hyperplane divisor and $F_{0}$ is a ruling plane of $S(1,1,1)$. Therefore $S$ is a surface of maximal sectional regularity and $\mathbb{F}(S)=S(1,1,1)$ (cf. Theorem 6.3(b)). It follows that

$$
Y \cap \mathbb{P}^{5}=S(1,1,1) \subset \mathbb{F}(X)
$$

for general $\mathbb{P}^{5} \in \mathbb{G}\left(5, \mathbb{P}^{n+3}\right)$ and hence $Y \subset \mathbb{F}(X)$. Then we get $Y=\mathbb{F}(X)$ from our classification result of $\mathbb{F}(X)$ in the present theorem.

Acknowledgement. The first named author thanks to the Korea University Seoul, to the Mathematisches Forschungsinstitut Oberwolfach, to the Martin-Luther Universität Halle and to the Deutsche Forschungsgemeinschaft for their hospitality and the financial support provided during the preparation of this work. The second named author was supported by the Nation Researcher program 2010-0020413 of NRF and MEST. The third named author was supported by the NRF-DAAD GEnKO Program (NRF-2011-0021014).

\section{REFERENCES}

[1] Bertin, M-A. : On the regularity of varieties having an extremal secant line. Journal für die reine und angewandte Mathematik. 545 (2002), 167-181.

[2] Brodmann, M., Lee, W., Park, E., Schenzel, P.: On surfaces of maximal sectional regularity. In preparation. 
[3] Brodmann, M., Schenzel, P.: On projective curves of maximal regularity. Mathematische Zeitschrift 244 (2003), 271-289.

[4] Brodmann, M., Schenzel, P.: Projective curves with maximal regularity and applications to syzygies and surfaces. Manuscripta Mathematica 135 (2011) 469-495.

[5] Castelnuovo, G.: Sui multipli di une serie lineare di gruppi di punti appartenente ad une curva algebraic, Rend. Circ. Mat. Palermo (2) 7 (1893), 89-110.

[6] Eisenbud, D., Gôto, S.: Linear free resolutions and minimal multiplicity. Journal of Algebra 88 (1984) 89-133.

[7] Ferraro, R.: Weil divisors on rational normal scrolls, Lecture Notes in Pure and Applied Mathematics, 217 (2001), 183-198.

[8] Flenner, H., O'Caroll, L., Vogel, W.: Joins and intersections. Springer Monographs in Mathematics, Springer Verlag, Berlin/Heidelberg/New York, 1990.

[9] Fujita, T.: Classification theories of polarized varieties. London Mathematical Society Lecture Notes Series 155, Cambridge University Press, 1990.

[10] Gruson, L., Lazarsfeld, R., Peskine, C.: On a theorem of Castelnuovo and the equations defining space curves. Inventiones Mathematicae 72 (1983), 491-506.

[11] Hoa, L-T., Stückrad, J., Vogel, W.: Towards a structure theory for projective varieties of degree=codimension+2. Journal of Pure and Applied Algebra 71 (1991), 203-231.

[12] Kleiman, S.: The transversality of a genearal translate. Compositio Mathematica 28 (1974) 287-297.

[13] Kwak, S., Park, E.: Some effects of property $N_{p}$ on the higher normality and defining equations of nonlinearly normal varieties. Journal für die reine und angewandte Mathematik 582 (2005) 87-105.

[14] Lazarsfeld, R.: A sharp Castelnuovo bound for smooth surfaces. Duke Mathematical Jornal 55 (1987) 423-429.

[15] Mumford, D.: Lectures on curves on an algebraic surface. Annals of Mathematics Studies 59, Princeton University Press, Princeton, 1966.

[16] Mryazaki, C.: Sharp Bounds on Castelnuovo-Mumford regularity. Transactions of the American Mathematical Society 352 (1999), 1675-1686.

[17] Noma, A.: Multisecant lines to projective varieties. Projective varieties with unexpected properties, 349 - 359, Walter de Gruyter GmbH and Co. KG, Berlin, 2005.

[18] PARK, E.: Smooth varieties of almost minimal degree. Journal of Algebra 314 (2007), 185-208.

[19] PARK, E.: On syzygies of divisors of rational normal scrolls. Mathematische Nachrichten 287, No. 11-12 (2014), 1383-1393.

[20] Pinkham, H.: A Castelnuovo bound for smooth surfaces. Inventiones Mathematicae 83 (1986), 321-332.

[21] Schreyer, F-O.: Syzygies of canonical curves and special linear series. Mathematische Annalen 275 (1986), 105-137.

Universität ZÜRICh, Institut fÜR MAthematik, Winterthurerstrasse 190, CH - Zürich, SWITZERLAND

E-mail address: brodmann@math.unizh.ch

Department of Applied Mathematics, Pukyong National University, Busan 608-737, KorEA

E-mail address: wslee@pknu.ac.kr

Korea University, Department of Mathematics, Anam-dong, Seongbuk-gu, Seoul 136701, Republic of KoreA

E-mail address: euisungpark@korea.ac.kr

Martin-Luther-Universität Halle-Wittenberg, Institut für Informatik, Von-SeckenDORfF-Platz 1, D - 06120 Halle (SaAle), Germany

E-mail address: schenzel@informatik.uni-halle.de 